Comment. Math. Helv. 79 (2004) 396-426 0010-2571/04/020396-31

DOI $10.1007 / \mathrm{s} 00014-003-0788-3$
(C) 2004 Birkhäuser Verlag, Basel

Commentarii Mathematici Helvetici

\title{
Varieties of pairs of nilpotent matrices annihilating each other
}

Jan Schröer

Abstract. We classify the irreducible components of the varieties

$$
\mathrm{V}(n, a, b)=\left\{(A, B) \in \mathrm{M}_{n}(K) \times \mathrm{M}_{n}(K) \mid A B=B A=A^{a}=B^{b}=0\right\} .
$$

Mathematics Subject Classification (2000). 14M99, 16 G10.

Keywords. Nilpotent matrix, irreducible component, Gelfand-Ponomarev algebra, string module, band module.

\section{Introduction and main results}

Let $\mathrm{M}_{n}(K)$ be the set of $n \times n$-matrices with entries in an algebraically closed field $K$. The study of affine varieties given by matrices or pairs of matrices, which satisfy certain relations, is a classical subject. One fundamental question is the decomposition of these varieties into irreducible components. Consider the varieties

$$
\mathrm{N}(n, l)=\left\{M \in \mathrm{M}_{n}(K) \mid M^{l}=0\right\}
$$

and

$$
\mathrm{Z}(n)=\left\{(A, B) \in \mathrm{M}_{n}(K) \times \mathrm{M}_{n}(K) \mid A B=B A=0\right\} .
$$

The variety $\mathrm{N}(n, l)$ is irreducible by $[\mathrm{Ge}]$ and $[\mathrm{H}]$, and the irreducible components of $\mathrm{Z}(n)$ are

$$
\{(A, B) \in \mathrm{Z}(n) \mid \operatorname{rk}(A) \leq n-i, \operatorname{rk}(B) \leq i\}
$$

for $0 \leq i \leq n$. For $n, a, b \geq 2$ define

$$
\begin{aligned}
\mathrm{V}(n, a, b) & =\left\{(A, B) \in \mathrm{M}_{n}(K) \times \mathrm{M}_{n}(K) \mid A B=B A=A^{a}=B^{b}=0\right\} \\
& =(\mathrm{N}(n, a) \times \mathrm{N}(n, b)) \cap \mathrm{Z}(n) .
\end{aligned}
$$

Our main result is the classification of irreducible components of $\mathrm{V}(n, a, b)$. This question appears for $a=b=n$ as an open problem in [K, Problem 3, p. 208]. In this special case, we get the following surprising result: 
Theorem 1.1. The irreducible components of $\mathrm{V}(n, n, n)$ are

$$
\{(A, B) \in \mathrm{V}(n, n, n) \mid \operatorname{rk}(A) \leq n-i, \operatorname{rk}(B) \leq i\}
$$

for $1 \leq i \leq n-1$. Each component has dimension $n^{2}-n+1$.

Thus each irreducible component of $\mathrm{V}(n, n, n)$ is the intersection of an irreducible component of $\mathrm{Z}(n)$ with $\mathrm{N}(n, n) \times \mathrm{N}(n, n)$. The case $a=b=2$ and $n$ arbitrary was studied in $[\mathrm{M}]$.

A partition of $n$ is a sequence $\mathbf{p}=\left(p_{1}, \cdots, p_{t}\right)$ of positive integers such that $\sum_{i=1}^{t} p_{i}=n$ and $p_{i} \geq p_{i+1}$ for all $i$. Let $l(\mathbf{p})=t$ be the length of $\mathbf{p}$. The set of partitions $\mathbf{p}$ of $n$ with $p_{i} \leq a$ for all $i$ is denoted by $\mathcal{P}(n, a)$.

By $\unlhd$ we denote the usual dominance order on $\mathcal{P}(n, a)$, see Section 5 for a definition.

The conjugacy classes of matrices in $\mathrm{N}(n, a)$ are parametrized by $\mathcal{P}(n, a)$. Namely, for a matrix $M \in \mathrm{N}(n, a)$, let $J(M)$ be its Jordan normal form, and set $p(M)=\left(p_{1}, \cdots, p_{t}\right)$ where the $p_{i}$ are the sizes of the Jordan blocks of $J(M)$, ordered decreasingly. Clearly, we have $p(M) \in \mathcal{P}(n, a)$. For $\mathbf{p} \in \mathcal{P}(n, a)$ let

$$
C(\mathbf{p})=\{M \in \mathrm{N}(n, a) \mid p(M)=\mathbf{p}\}
$$

be the corresponding conjugacy class in $\mathrm{N}(n, a)$.

There are two projection maps

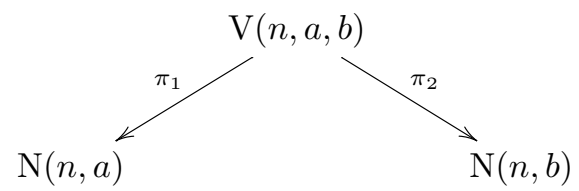

where $\pi_{1}(A, B)=A$ and $\pi_{2}(A, B)=B$. For $\mathbf{a} \in \mathcal{P}(n, a)$ let

$$
\Delta(\mathbf{a})=\pi_{1}^{-1}(C(\mathbf{a})) .
$$

In general, $\Delta(\mathbf{a})$ is not irreducible. Only if $a=b=n$, these sets have nice properties:

Theorem 1.2. For each $\mathbf{a} \in \mathcal{P}(n, n)$ the set $\Delta(\mathbf{a}) \subset \mathrm{V}(n, n, n)$ is locally closed and irreducible. We have

$$
\Delta(1, \cdots, 1) \subset \overline{\Delta(2,1, \cdots, 1)},
$$

and if $\mathbf{a} \neq(1, \cdots, 1)$, then

$$
\Delta(\mathbf{a}) \subset \overline{\Delta(\mathbf{b})}
$$

if and only if $\mathbf{a} \unlhd \mathbf{b}$ and $l(\mathbf{a})=l(\mathbf{b})$.

For the study of the general case, define the standard stratification of $\mathrm{V}(n, a, b)$ as follows: Let

$$
\mathcal{P}(n, a, b)=\mathcal{P}(n, a) \times \mathcal{P}(n, b)
$$


For $(\mathbf{a}, \mathbf{b}) \in \mathcal{P}(n, a, b)$ let

$$
\Delta(\mathbf{a}, \mathbf{b})=\pi_{1}^{-1}(C(\mathbf{a})) \cap \pi_{2}^{-1}(C(\mathbf{b}))
$$

be the corresponding stratum of the standard stratification. Unfortunately, these strata are in general not very well-behaved:

- A stratum might be empty;

- Strata are not necessarily irreducible;

- The closure of a stratum is in general not a union of strata.

However, the socalled 'regular strata' have nice properties. Observe that for $(A, B) \in \mathrm{V}(n, a, b)$ the inequality

$$
\operatorname{rk}(A)+\operatorname{rk}(B) \leq n
$$

holds. This follows already from the condition $A B=0$. We call $(A, B)$ regular if $\operatorname{rk}(A)+\operatorname{rk}(B)=n$. An irreducible component of $\mathrm{V}(n, a, b)$ is regular if it contains a regular element, and we call $(\mathbf{a}, \mathbf{b}) \in \mathcal{P}(n, a, b)$ and also its corresponding stratum $\Delta(\mathbf{a}, \mathbf{b})$ regular if $\Delta(\mathbf{a}, \mathbf{b})$ contains a regular element.

For a partition $\mathbf{p}=\left(p_{1}, \cdots, p_{t}\right) \neq(1, \cdots, 1)$ define

$$
\mathbf{p}-1=\left(p_{1}-1, \cdots, p_{r}-1\right)
$$

where $r=\max \left\{1 \leq i \leq t \mid p_{i} \geq 2\right\}$. For example,

$$
(3,2,2,1)-1=(2,1,1)
$$

The following result determines which strata are regular.

Proposition 1.3. For $(\mathbf{a}, \mathbf{b}) \in \mathcal{P}(n, a, b)$ the following are equivalent:

(1) $(\mathbf{a}, \mathbf{b})$ is regular;

(2) $l(\mathbf{a})+l(\mathbf{b})=n$ and $l(\mathbf{a}-1)=l(\mathbf{b}-1)$.

In this case, all elements in $\Delta(\mathbf{a}, \mathbf{b})$ are regular.

If $\mathbf{p}$ is a partition, then let

$$
|i \in \mathbf{p}|
$$

be the number of entries of $\mathbf{p}$ which are equal to $i$. The next theorem yields a classification of all regular irreducible components.

Theorem 1.4. If $(\mathbf{a}, \mathbf{b}) \in \mathcal{P}(n, a, b)$ is regular, then $\Delta(\mathbf{a}, \mathbf{b})$ is locally closed and irreducible. In this case, the closure of $\Delta(\mathbf{a}, \mathbf{b})$ is an irreducible component of $\mathrm{V}(n, a, b)$ if and only if the following hold:

(1) a has at most one entry different from 1,2 and a;

(2) $\mathbf{b}$ has at most one entry different from 1,2 and $b$;

(3) $l(\mathbf{a}-1) \leq|a \in \mathbf{a}|+|b \in \mathbf{b}|+1$.

Next, we determine when all irreducible components of $\mathrm{V}(n, a, b)$ are regular. 
Proposition 1.5. The set of regular elements is dense in $\mathrm{V}(n, a, b)$ if and only if $n \leq a+b-2$ or $n=a+b$.

The classification of the non-regular irreducible components of $\mathrm{V}(n, a, b)$ is more complicated and needs more notation. We state and prove the result in Section 8.

The paper is organized as follows: In Section 2 we repeat some basics on varieties of modules. In particular, we recall Richmond's construction of a stratification of these varieties, which we will use throughout. We regard $\mathrm{V}(n, a, b)$ as a variety of modules over a Gelfand-Ponomarev algebra, and we use module theory to classify the irreducible components of $\mathrm{V}(n, a, b)$. Section 3 is a collection of mostly known results on Gelfand-Ponomarev algebras. Richmond's stratification turns out to be finite for $\mathrm{V}(n, a, b)$. This is studied in Section 4. In Section 5 we prove that all regular strata are irreducible. This is used in Section 6 to prove Theorem 1.1. The classification of all regular components of $\mathrm{V}(n, a, b)$ can be found in Section 7. Theorem 1.2 is proved at the end of Section 7. The main result of Section 8 is the classification of all non-regular components of $\mathrm{V}(n, a, b)$. Finally, some examples are given in Section 9 .

Acknowledgements. The author received a Postdoctoral Fellowship from the DAAD, Germany, for a stay at the UNAM in Mexico City, where most of this work was done. He thanks Christof Geiß and Lutz Hille for helpful and interesting discussions.

\section{Varieties of modules}

Let $A$ be a finitely generated $K$-algebra. Fix a set $a_{1}, \cdots, a_{N}$ of generators of $A$. $\operatorname{By} \bmod (A, n)$ we denote the affine variety of $A$-module structures on $K^{n}$. Each such $A$-module structure corresponds to a $K$-algebra homomorphism $A \rightarrow \mathrm{M}_{n}(K)$, or equivalently to a tuple $\left(M_{1}, \cdots, M_{N}\right)$ of $n \times n$-matrices such that the $M_{i}$ satisfy the same relations as the $a_{i}$. The group $\mathrm{GL}_{n}(K)$ acts by simultaneous conjugation on $\bmod (A, n)$, and the orbits of this action are in 1-1 correspondence with the isomorphism classes of $n$-dimensional $A$-modules. An orbit $\mathcal{O}(X)$ of a module $X$ has dimension $n^{2}-\operatorname{dim} \operatorname{End}_{A}(X)$. If $\mathcal{O}(X)$ is contained in the closure of an orbit $\mathcal{O}(Y)$, then we write $Y \leq_{\operatorname{deg}} X$. It is well known that $Y \leq_{\operatorname{deg}} X$ implies $\operatorname{dim} \operatorname{Hom}_{A}(Y, M) \leq \operatorname{dim} \operatorname{Hom}_{A}(X, M)$ for all modules $M$, see for example [Bo]. If

$$
0 \longrightarrow X \longrightarrow Y \longrightarrow Z \longrightarrow 0
$$

is a short exact sequence, then $Y \leq_{\operatorname{deg}} X \oplus Z$. If there exists a module $Z$ and a short exact sequence

$$
0 \longrightarrow X \longrightarrow Y \oplus Z \longrightarrow Z \longrightarrow 0
$$


then it is proved in [Rie] that $Y \leq_{\operatorname{deg}} X$. The converse is also true by [Z]. Short exact sequences of this form are called Riedtmann sequences. We call a module $X$ a minimal degeneration if there exists no module $Y$ with $Y<_{\operatorname{deg}} X$.

Now, let $A$ be a finite-dimensional $K$-algebra, and let $\mathcal{I}_{A}(n)$ be a set of representatives of isomorphism classes of submodules of $A^{n}$ which have dimension $n(d-1)$ where $d=\operatorname{dim}(A)$. The modules in $\mathcal{I}_{A}(n)$ are called the index modules of $A$. For each $L \in \mathcal{I}_{A}(n)$ let $\mathcal{S}(L)$ be the set of points $X \in \bmod (A, n)$ such that there exists a short exact sequence

$$
0 \longrightarrow L \longrightarrow A^{n} \longrightarrow X \longrightarrow 0
$$

of $A$-modules. Such a set $\mathcal{S}(L)$ is called a stratum. Note that $\bmod (A, n)$ is the disjoint union of the $\mathcal{S}(L)$ where $L$ runs through $\mathcal{I}_{A}(n)$. The following theorem can be found in $[R]$.

Theorem 2.1 (Richmond). Let $A$ be a finite-dimensional $K$-algebra. Then the following hold:

(1) For each $L \in \mathcal{I}_{A}(n)$ the stratum $\mathcal{S}(L)$ is smooth, locally closed, irreducible and has dimension

$$
\operatorname{dim} \operatorname{Hom}_{A}\left(L, A^{n}\right)-\operatorname{dim} \operatorname{End}_{A}(L) ;
$$

(2) Let $L, M \in \mathcal{I}_{A}(n)$. If $\mathcal{S}(L)$ is contained in the closure of $\mathcal{S}(M)$, then $M \leq_{\operatorname{deg}} L$

(3) Let $L, M \in \mathcal{I}_{A}(n)$. If $M \leq_{\operatorname{deg}} L$ and

$$
\operatorname{dim} \operatorname{Hom}_{A}(L, A)=\operatorname{dim} \operatorname{Hom}_{A}(M, A),
$$

then $\mathcal{S}(L)$ is contained in the closure of $\mathcal{S}(M)$.

Unfortunately, the converse of the second part of this theorem is usually wrong. So it remains a difficult problem to decide when a stratum is contained in the closure of another stratum. Another problem is, that the set $\mathcal{I}_{A}(n)$ is often infinite. Following $[\mathrm{R}]$ an algebra $A$ is called subfinite if $\mathcal{I}_{A}(n)$ is finite for all $n$.

\section{Gelfand-Ponomarev algebras}

We identify $\mathrm{V}(n, a, b)$ with the variety of $n$-dimensional modules over the algebra

$$
\Lambda=\Lambda_{a, b}=K[x, y] /\left(x y, x^{a}, y^{b}\right) .
$$

We call $\Lambda$ a Gelfand-Ponomarev algebra.

The group $\mathrm{GL}_{n}(K)$ acts on $\mathrm{V}(n, a, b)=\bmod (\Lambda, n)$ by simultaneous conjugation, i.e.

$$
g \cdot(A, B)=\left(g A g^{-1}, g B g^{-1}\right)
$$


The orbits of this action are in 1-1 correspondence with the isomorphism classes of $n$-dimensional $\Lambda$-modules. By $\mathcal{O}(M)$ we denote the orbit of an element $M \in$ $\mathrm{V}(n, a, b)$.

In the following we repeat Gelfand and Ponomarev's classification of indecomposable $\Lambda$-modules (by a 'module' we always mean a finite-dimensional right module). As a main reference we use [GP], but see also [BR].

A string of length $n \geq 1$ is a word $c_{1} \cdots c_{n}$ with letters $c_{i} \in\{x, y\}$ such that no subword is of the form $x^{a}$ or $y^{b}$. Additionally, we define a string 1 of length 0 . Set $x^{0}=y^{0}=1$.

The length of an arbitrary string $C$ is denoted by $|C|$. Let $C=c_{1} \cdots c_{n}$ and $D=d_{1} \cdots d_{m}$ be strings of length at least one. If $C D=c_{1} \cdots c_{n} d_{1} \cdots d_{m}$ is a string, then we say that the concatenation of $C$ and $D$ is defined. For an arbitrary string $C$ let $1 C=C 1=C$.

For each string $C$ we construct a string module $M(C)$ over $\Lambda$ as follows: First, assume that $n=|C| \geq 1$. Fix a basis $\left\{z_{1}, \cdots, z_{n+1}\right\}$ of $M(C)$. Given an arrow $\alpha \in\{x, y\}$ let

$$
z_{i} \cdot \alpha= \begin{cases}z_{i+1} & \text { if } \alpha=c_{i}=y \text { and } 1 \leq i \leq n \\ z_{i-1} & \text { if } \alpha=c_{i-1}=x \text { and } 2 \leq i \leq n+1 \\ 0 & \text { otherwise. }\end{cases}
$$

For $C=1$ let $S=M(C)$ be the one-dimensional module with basis $\left\{z_{1}\right\}$ such that $z_{1} \cdot x=z_{1} \cdot y=0$. This is the unique simple $\Lambda$-module. The $z_{i}$ are called the canonical basis vectors of $M(C)$.

For example, let $C=x x y x y$. Then the string module $M(C)$ looks as in Figure 1 , where $z_{1}, \cdots, z_{6}$ are the canonical basis vectors of $M(C)$, and the arrows indicate how the generators $x$ and $y$ of $\Lambda$ operate on these basis vectors. Set $(A, B)=$

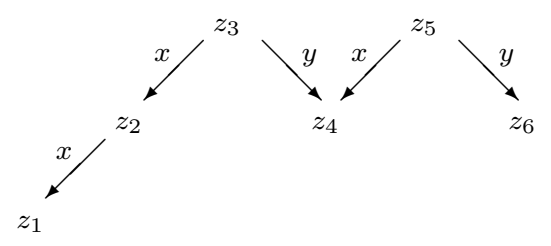

FIG. 1. The string module $M(x x y x y)$

$M(x x y x y)$. We have

$$
(A, B) \in \pi_{1}^{-1}(C(3,2,1)) \cap \pi_{2}^{-1}(C(2,2,1,1))=\Delta((3,2,1),(2,2,1,1)) .
$$

A string $C=c_{1} \cdots c_{n}$ of length at least one is called a band if all powers $C^{m}$ are defined. Next, we associate to a given band $B=b_{1} \cdots b_{m}$ and some $n \geq 1$ a family

$$
\left\{M\left(B, \lambda_{1}, \cdots, \lambda_{n}\right) \mid \lambda_{i} \in K^{*}, 1 \leq i \leq n\right\}
$$


of band modules. Fix a basis $\left\{z_{1 j}, \cdots, z_{m j} \mid 1 \leq j \leq n\right\}$ of $M\left(B, \lambda_{1}, \cdots, \lambda_{n}\right)$. For $\alpha \in\{x, y\}$ define

$$
z_{1 j} \cdot \alpha= \begin{cases}z_{2 j} & \text { if } \alpha=b_{1}=y \\ \lambda_{j} z_{m j}+z_{m j-1} & \text { if } \alpha=b_{m}=x \text { and } 2 \leq j \leq n, \\ \lambda_{1} z_{m 1} & \text { if } \alpha=b_{m}=x \text { and } j=1, \\ 0 & \text { otherwise }\end{cases}
$$

and let

$$
z_{m j} \cdot \alpha= \begin{cases}z_{m-1 j} & \text { if } \alpha=b_{m-1}=x, \\ \lambda_{j} z_{1 j}+z_{1 j-1} & \text { if } \alpha=b_{m}=y \text { and } 2 \leq j \leq n, \\ \lambda_{1} z_{11} & \text { if } \alpha=b_{m}=y \text { and } j=1, \\ 0 & \text { otherwise. }\end{cases}
$$

For $2 \leq i \leq m-1$ and $1 \leq j \leq n$ we define

$$
z_{i j} \cdot \alpha= \begin{cases}z_{i+1 j} & \text { if } \alpha=b_{i}=y \\ z_{i-1 j} & \text { if } \alpha=b_{i-1}=x, \\ 0 & \text { otherwise. }\end{cases}
$$

The $z_{i j}$ are called the canonical basis vectors of $M\left(B, \lambda_{1}, \cdots, \lambda_{n}\right)$.

For example, let $B=x x y x y$. Then the band module $M\left(B, \lambda_{1}, \lambda_{2}\right)$ looks as in Figure 2. The arrows in Figure 2 indicate how the generators $x$ and $y$ of $\Lambda$ operate

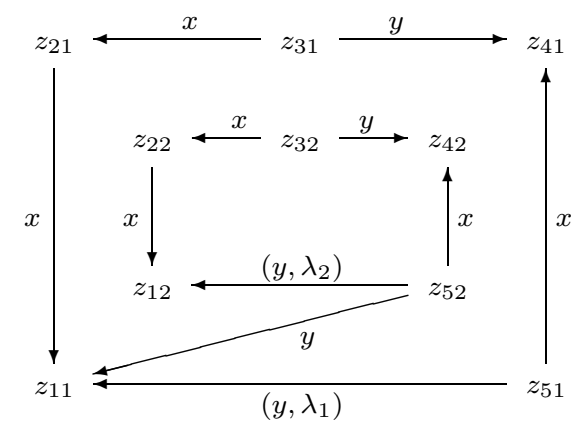

FIG. 2. The band module $M\left(x x y x y, \lambda_{1}, \lambda_{2}\right)$

on the canonical basis vectors of $M\left(B, \lambda_{1}, \lambda_{2}\right)$. For example, $z_{51} \cdot y=\lambda_{1} z_{11}$, $z_{52} \cdot y=\lambda_{2} z_{12}+z_{11}, z_{32} \cdot y=z_{42}$ etc.

The next lemma is proved by straightforward base change calculations.

Lemma 3.1. Let $M\left(B, \lambda_{1}, \cdots, \lambda_{n}\right)$ be a band module. If $\lambda_{l} \neq \lambda_{l+1}$ for some $l$, then $M\left(B, \lambda_{1}, \cdots, \lambda_{n}\right)$ is isomorphic to

$$
M\left(B, \lambda_{1}, \cdots, \lambda_{l}\right) \oplus M\left(B, \lambda_{l+1}, \cdots, \lambda_{n}\right) .
$$



$[\mathrm{BR}]$.

If $\lambda_{i}=\lambda_{j}$ for all $i$ and $j$, then define $M(B, \lambda, n)=M\left(B, \lambda_{1}, \cdots, \lambda_{n}\right)$, compare BR].

A band $B$ is called periodic if there exists some string $C$ such that $B=C^{m}$ for some $m \geq 2$. A band is called primitive if it is not periodic. For primitive bands $B_{1}$ and $B_{2}$ define $B_{1} \sim B_{2}$ if $B_{1}=B B^{\prime}$ and $B_{2}=B^{\prime} B$ for some strings $B$ and $B^{\prime}$. Let $\mathcal{S}$ be the set of strings, and let $\mathcal{B}$ be a set of representatives of equivalence classes of primitive bands with respect to the equivalence relation $\sim$. The following theorem is proved in $[\mathrm{GP}]$.

Theorem 3.2 (Gelfand-Ponomarev). The modules $M(C)$ and $M(B, \lambda, n)$ with $C \in \mathcal{S}, B \in \mathcal{B}, \lambda \in K^{*}$ and $n \geq 1$ is a complete set of representatives of isomorphism classes of indecomposable $\Lambda$-modules.

The next lemma follows from the construction of string and band modules and from Theorem 3.2.

Lemma 3.3. If $(A, B) \in \mathrm{V}(n, a, b)$, then

$$
n-s=\operatorname{rk}(A)+\operatorname{rk}(B),
$$

where $s$ is the number of string modules in a decomposition of $(A, B)$ into a direct sum of indecomposable modules.

Corollary 3.4. An element in $\mathrm{V}(n, a, b)$ is regular if and only if it is isomorphic to a direct sum of band modules.

Let $B_{1}, \cdots, B_{m}$ be bands. For positive integers $p_{1}, \cdots, p_{m}$ set

$$
\begin{aligned}
p & =\sum_{i=1}^{m} p_{i}, \\
n & =\sum_{i=1}^{m} p_{i}\left|B_{i}\right|, \\
F^{p} & =\left\{\left(\lambda_{1}, \cdots, \lambda_{p}\right) \in K^{p} \mid \lambda_{i} \neq \lambda_{j} \neq 0 \text { for all } i \neq j\right\} .
\end{aligned}
$$

Define a morphism of varieties

$$
\begin{gathered}
\mathrm{GL}_{n}(K) \times F^{p} \longrightarrow \mathrm{V}(n, a, b) \\
\left(g,\left(\lambda_{11}, \cdots, \lambda_{p_{1} 1}, \cdots, \lambda_{1 m}, \cdots, \lambda_{p_{m} m}\right)\right) \mapsto g \cdot\left(\bigoplus_{j=1}^{m} M\left(B_{j}, \lambda_{1 j}, \cdots, \lambda_{p_{j} j}\right)\right) .
\end{gathered}
$$

The image of this morphism is denoted by

$$
\mathcal{F}=\mathcal{F}\left(\left(B_{1}, p_{1}\right), \cdots,\left(B_{m}, p_{m}\right)\right) .
$$


We say that $\mathcal{F}$ is a $p$-parametric family. In case $p_{i}=1$ for some $i$, we write also just $B_{i}$ instead of $\left(B_{i}, p_{i}\right)$. It follows from $[\mathrm{Kr}]$ that $\operatorname{dim} \mathcal{O}(y)$ is constant for all $y$ in a given family $\mathcal{F}$. The following lemma is straightforward.

Lemma 3.5. Any p-parametric family $\mathcal{F}$ is constructible, irreducible and has dimension $p+\operatorname{dim} \mathcal{O}(y)$ where $y$ is any point in $\mathcal{F}$.

Lemma 3.6. Each direct sum of band modules is contained in the closure of some family $\mathcal{F}$.

Proof. A band module $M(B, \lambda, n)$ is obviously contained in the closure of the set of all band modules $M\left(B, \lambda_{1}, \cdots, \lambda_{n}\right)$ where the $\lambda_{i}$ are pairwise different.

For a string $C$ define

$$
\mathcal{P}(C)=\{(D, E, F) \mid D, E, F \in \mathcal{S} \text { and } D E F=C\} .
$$

We call $(D, E, F) \in \mathcal{P}(C)$ a factor string of $C$ if the following hold:

(1) Either $D=1$ or $D=d_{1} \cdots d_{n}$ where $d_{n}=x$;

(2) Either $F=1$ or $F=f_{1} \cdots f_{m}$ where $f_{1}=y$.

Dually, we call $(D, E, F)$ a substring of $C$ if the following hold:

(1) Either $D=1$ or $D=d_{1} \cdots d_{n}$ where $d_{n}=y$;

(2) Either $F=1$ or $F=f_{1} \cdots f_{m}$ where $f_{1}=x$.

Let $\operatorname{fac}(C)$ be the set of factor strings of $C$, and by $\operatorname{sub}(C)$ we denote the set of substrings of $C$. For strings $C_{1}$ and $C_{2}$ let

$$
\mathcal{A}\left(C_{1}, C_{2}\right)=\left\{\left(\left(D_{1}, E_{1}, F_{1}\right),\left(D_{2}, E_{2}, F_{2}\right)\right) \in \operatorname{fac}\left(C_{1}\right) \times \operatorname{sub}\left(C_{2}\right) \mid E_{1}=E_{2}\right\} .
$$

For example, if $C_{1}=x x y$ and $C_{2}=x y x x$, then

$$
\begin{aligned}
\mathcal{A}\left(C_{1}, C_{2}\right) & =\{((x x, 1, y),(1,1, x y x x)),((x x, 1, y),(x y, 1, x x)), \\
& ((1, x x, y),(x y, x x, 1)),((x, x, y),(x y, x, x)),((x, x y, 1),(1, x y, x x))\} .
\end{aligned}
$$

For each $a=\left(\left(D_{1}, E_{1}, F_{1}\right),\left(D_{2}, E_{2}, F_{2}\right)\right) \in \mathcal{A}\left(C_{1}, C_{2}\right)$ we define a homomorphism

$$
f_{a}: M\left(C_{1}\right) \longrightarrow M\left(C_{2}\right)
$$

as follows: Define

$$
f_{a}\left(z_{\left|D_{1}\right|+i}\right)=z_{\left|D_{2}\right|+i}
$$

for $1 \leq i \leq\left|E_{1}\right|+1$, and all other canonical basis vectors of $M\left(C_{1}\right)$ are mapped to 0 . Such homomorphisms are called graph maps. The following theorem is a special case of the main result in $[\mathrm{CB}]$.

Theorem 3.7 (Crawley-Boevey). The graph maps $\left\{f_{a} \mid a \in \mathcal{A}\left(C_{1}, C_{2}\right)\right\}$ form a $K$-basis of the homomorphism space $\operatorname{Hom}_{\Lambda}\left(M\left(C_{1}\right), M\left(C_{2}\right)\right)$. 
There is the following multiplicative behaviour of graph maps: Let $f_{a}: M\left(C_{1}\right)$ $\rightarrow M\left(C_{2}\right)$ and $f_{b}: M\left(C_{2}\right) \rightarrow M\left(C_{3}\right)$ be graph maps. Then the composition $f_{a} f_{b}: M\left(C_{1}\right) \rightarrow M\left(C_{3}\right)$ is either 0 or a graph map.

\section{Index modules of Gelfand-Ponomarev algebras}

A module $M$ is called biserial if it is isomorphic to

$$
\bigoplus_{i=1}^{m} M\left(x^{i} y^{j}\right)
$$

where $0 \leq i \leq a-1$ and $0 \leq j \leq b-1$. For example, $\Lambda$ regarded as a module over itself is isomorphic to the biserial module $M\left(x^{a-1} y^{b-1}\right)$. Note also that any projective $\Lambda$-module is isomorphic to $\Lambda^{n}$ for some $n \geq 1$.

Lemma 4.1. Gelfand-Ponomarev algebras are subfinite, and all their index modules are biserial.

Proof. Any Gelfand-Ponomarev algebra $\Lambda$ is a monomial algebra. Thus by $[\mathrm{ZH}$, Lemma 3], if $U$ is a submodule of a projective $\Lambda$-module, then

$$
(U \cdot x) \cap(U \cdot y)=0 .
$$

It follows from the description of indecomposable $\Lambda$-modules that the biserial modules are the only $\Lambda$-modules which have this property.

A case by case analysis shows the following:

Lemma 4.2. A biserial $\Lambda$-module

$$
L=S^{m_{s}} \oplus \bigoplus_{i \geq 1} M\left(x^{i}\right)^{m_{x i}} \oplus \bigoplus_{j \geq 1} M\left(y^{j}\right)^{m_{y j}} \oplus \bigoplus_{i, j \geq 1} M\left(x^{i} y^{j}\right)^{m_{i j}}
$$

is isomorphic to a submodule of $\Lambda^{n}$ if and only if the following hold:

$$
\begin{aligned}
m_{x i} \neq 0 & \Longrightarrow i \leq a-2, \\
m_{y j} \neq 0 & \Longrightarrow j \leq b-2, \\
m_{i b-1} \neq 0 & \Longrightarrow i=a-1, \\
m_{a-1 j} \neq 0 & \Longrightarrow j=b-1,
\end{aligned}
$$




$$
\begin{aligned}
\sum_{i \geq 1} m_{x i}+\sum_{i, j \geq 1} m_{i j} & \leq n, \\
\sum_{j \geq 1} m_{y j}+\sum_{i, j \geq 1} m_{i j} & \leq n, \\
m_{s}+\sum_{i \geq 1} m_{x i}+\sum_{j \geq 1} m_{y j}+2\left(\sum_{i, j \geq 1} m_{i j}\right) & \leq 2 n .
\end{aligned}
$$

The dimension of $L$ is

$$
m_{s}+\sum_{i \geq 1} m_{x i}(i+1)+\sum_{j \geq 1} m_{y j}(j+1)+\sum_{i, j \geq 1} m_{i j}(i+j+1) .
$$

Lemma 4.3. Let $L \in \mathcal{I}_{\Lambda}(n)$ and assume that $L$ is the direct sum of $m$ indecomposable modules. Let $p$ be the number of indecomposable projective modules among these direct summands. Then we have

$$
\operatorname{dim} \operatorname{Hom}(L, \Lambda)=n(d-1)+m-p
$$

where $d=\operatorname{dim}(\Lambda)$.

Proof. We have $\operatorname{dim} \operatorname{Hom}(\Lambda, \Lambda)=\operatorname{dim}(\Lambda)=d$ and so $\operatorname{dim} \operatorname{Hom}(P, \Lambda)=\operatorname{dim}(P)$ for any projective module $P$. Each indecomposable non-projective direct summand of $L$ is of the form $M\left(x^{i} y^{j}\right)$ with $0 \leq i \leq a-2$ and $0 \leq j \leq b-2$. We have

$$
\begin{aligned}
\operatorname{dim} M\left(x^{i} y^{j}\right) & =i+j+1, \\
\operatorname{dim} \operatorname{Hom}\left(M\left(x^{i} y^{j}\right), \Lambda\right) & =i+j+2 .
\end{aligned}
$$

This can be checked directly or by applying $[\mathrm{CB}]$. Since $\operatorname{dim}(L)=n(d-1)$, the result follows.

Lemma 4.4. If $0 \leq p \leq i \leq a-1$ and $0 \leq q \leq j \leq b-1$, then

$$
M\left(x^{i} y^{q}\right) \oplus M\left(x^{p} y^{j}\right) \leq_{\operatorname{deg}} M\left(x^{i} y^{j}\right) \oplus M\left(x^{p} y^{q}\right) .
$$

Proof. One can easily construct a short exact sequence

$$
0 \longrightarrow M\left(x^{i} y^{j}\right) \longrightarrow M\left(x^{i} y^{q}\right) \oplus M\left(x^{p} y^{j}\right) \longrightarrow M\left(x^{p} y^{q}\right) \longrightarrow 0 .
$$

A degeneration of the same form as in the previous lemma is called a flip degeneration. (We 'flip' $q$ and $j$.) An index module $L$ is called flip minimal if it is isomorphic to a direct sum of the form

$$
\Lambda^{p} \oplus \bigoplus_{i=1}^{t} M\left(x^{c_{i}} y^{d_{t-i+1}}\right)
$$


such that $c_{i} \geq c_{i+1}, d_{i} \geq d_{i+1}, 0 \leq c_{i} \leq a-2$ and $0 \leq d_{i} \leq b-2$ for all $i$. It follows from the previous two lemmas that for any index module $L$ there exists a chain

$$
L_{1} \leq_{\operatorname{deg}} L_{2} \leq_{\text {deg }} \cdots \leq_{\text {deg }} L_{t}=L
$$

of flip degenerations of index modules with $L_{1}$ being flip minimal and

$$
\operatorname{dim} \operatorname{Hom}\left(L_{i}, \Lambda\right)=\operatorname{dim} \operatorname{Hom}\left(L_{1}, \Lambda\right)
$$

for all $i$.

Lemma 4.5. If $1 \leq p \leq i \leq a-2$ and $0 \leq q, j \leq b-1$, then

$$
M\left(x^{i+1} y^{j}\right) \oplus M\left(x^{p-1} y^{q}\right) \leq_{\operatorname{deg}} M\left(x^{i} y^{j}\right) \oplus M\left(x^{p} y^{q}\right) .
$$

Proof. One can construct a short exact sequence

$$
0 \longrightarrow M\left(x^{p} y^{q}\right) \longrightarrow M\left(x^{i+1} y^{j}\right) \oplus M\left(x^{p-1} y^{q}\right) \longrightarrow M\left(x^{i} y^{j}\right) \longrightarrow 0 .
$$

A degeneration of the same form as in the above lemma is called a box move degeneration. The modules over $K[x] /\left(x^{n}\right)$ correspond to partitions, or equivalently to Young diagrams, and the degenerations of these modules are given by moving boxes of the Young diagrams. We are in a similar situation here. Note that Lemma 4.5 has an obvious dual version, exchanging the roles of $x$ and $y$.

\section{Regular strata are irreducible}

For a partition $\mathbf{p}=\left(p_{1}, \cdots, p_{t}\right)$ let $Y(\mathbf{p})$ be its corresponding Young diagram, which has $p_{i}$ boxes in the $i$ th column. For example, the Young diagram $Y(3,2,2,1)$ looks as in Figure 3. For a partition $\mathbf{p}=\left(p_{1}, \cdots, p_{t}\right)$ the dual partition is defined

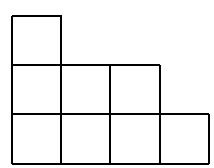

Fig. 3. The Young diagram $Y(3,2,2,1)$

as $\mathbf{p}^{*}=\left(r_{1}, \cdots, r_{p_{1}}\right)$, where the $r_{j}$ are the number of boxes in the rows of the Young diagram $Y(\mathbf{p})$, ordered decreasingly. For example,

$$
(3,2,2,1)^{*}=(4,3,1) .
$$

Now let $A \in \mathrm{N}(n, a)$ with $\mathbf{p}=p(A)$. Then the boxes of the Young diagram $Y(\mathbf{p})$ can be considered as a certain basis of $K^{n}$, and $A$ can be considered as an endomorphism of $K^{n}$. If $b$ is a box such that there is a box $b^{\prime}$ below $b$, then 
$A$ maps $b$ to $b^{\prime}$, and $b$ is mapped to 0 , otherwise. Figure 4 illustrates this for $p(A)=(3,2,2,1)$, where the arrows indicate how $A$ acts on the boxes. Now let

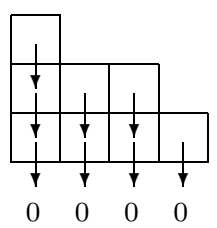

FIG. 4

$\mathbf{p}^{*}=\left(r_{1}, \cdots, r_{m}\right)$ be the dual partition of $\mathbf{p}$. Then $r_{1}=\operatorname{dim} \operatorname{Ker}(A), r_{1}+r_{2}=$ $\operatorname{dim} \operatorname{Ker}\left(A^{2}\right)$ etc.

If $\mathbf{p}$ and $\mathbf{q}$ are arbitrary partitions, then define $\mathbf{p} \unlhd \mathbf{q}$ if

$$
\sum_{i=1}^{l} p_{i} \leq \sum_{j=1}^{l} q_{j}
$$

for all $l$, where we set $p_{i}=0$ and $q_{j}=0$ for all $i>l(\mathbf{p})$ and $j>l(\mathbf{q})$. This partial order is usually called the dominance order. The proof of the following proposition can be found in $[\mathrm{Ge}]$, see also $[\mathrm{H}]$.

Proposition 5.1. For $\mathbf{p} \in \mathcal{P}(n, l)$ we have

$$
\operatorname{dim} C(\mathbf{p})=n^{2}-\sum_{i=1}^{t} r_{i}^{2}
$$

where $\mathbf{p}^{*}=\left(r_{1}, \cdots, r_{t}\right)$,

$$
C(\mathbf{p})=\left\{A \in \mathrm{N}(n, l) \mid \operatorname{rk}\left(A^{k}\right)=n-\sum_{j=1}^{k} r_{j}, 1 \leq k \leq t\right\}
$$

and

$$
\overline{C(\mathbf{p})}=\left\{A \in \mathrm{N}(n, l) \mid \operatorname{rk}\left(A^{k}\right) \leq n-\sum_{j=1}^{k} r_{j}, 1 \leq k \leq t\right\} .
$$

In particular, if $\mathbf{p}, \mathbf{q} \in \mathcal{P}(n, l)$, then $C(\mathbf{p}) \subseteq \overline{C(\mathbf{q})}$ if and only if $\mathbf{p} \unlhd \mathbf{q}$.

Recall that we defined two maps

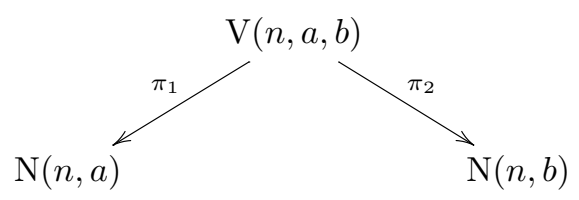


with $\pi_{1}(A, B)=A$ and $\pi_{2}(A, B)=B$, and for $(\mathbf{a}, \mathbf{b}) \in \mathcal{P}(n, a, b)$ we set

$$
\begin{aligned}
\Delta(\mathbf{a}) & =\pi_{1}^{-1}(C(\mathbf{a})), \\
\Delta(\mathbf{a}, \mathbf{b}) & =\pi_{1}^{-1}(C(\mathbf{a})) \cap \pi_{2}^{-1}(C(\mathbf{b})) .
\end{aligned}
$$

Thus, as a consequence of Proposition 5.1 we get

$$
\Delta(\mathbf{a})=\left\{(A, B) \in \mathrm{V}(n, a, b) \mid \operatorname{rk}\left(A^{k}\right)=n-\sum_{j=1}^{k} m_{j}, 1 \leq k \leq r\right\}
$$

and

$$
\begin{aligned}
\Delta(\mathbf{a}, \mathbf{b})=\left\{(A, B) \in \mathrm{V}(n, a, b) \mid \operatorname{rk}\left(A^{k}\right)=n-\right. & \sum_{j=1}^{k} m_{j}, 1 \leq k \leq r, \\
& \left.\operatorname{rk}\left(B^{l}\right)=n-\sum_{j=1}^{l} n_{j}, 1 \leq l \leq s\right\}
\end{aligned}
$$

where $\mathbf{a}^{*}=\left(m_{1}, \cdots, m_{r}\right)$ and $\mathbf{b}^{*}=\left(n_{1}, \cdots, n_{s}\right)$. In particular, $\Delta(\mathbf{a})$ and $\Delta(\mathbf{a}, \mathbf{b})$ are locally closed in $\mathrm{V}(n, a, b)$.

The following lemma is an easy exercise.

Lemma 5.2. If $M \in \mathrm{N}(n, l)$, then $\operatorname{rk}(M)=n-l(p(M))$.

Proof of Proposition 1.3. Let $(A, B) \in \mathrm{V}(n, a, b)$, and set

$$
(\mathbf{a}, \mathbf{b})=(p(A), p(B)) \in \mathcal{P}(n, a, b) .
$$

Assume that $(A, B)$ is regular, i.e. $\operatorname{rk}(A)+\operatorname{rk}(B)=n$. By Lemma 5.2 this is equivalent to $n=l(\mathbf{a})+l(\mathbf{b})$. Thus, if $\Delta(\mathbf{a}, \mathbf{b})$ contains a regular element, then all elements in $\Delta(\mathbf{a}, \mathbf{b})$ are regular. We know that $(A, B)$ is isomorphic to a direct sum of band modules. But any band is (up to equivalence) of the form $x^{c_{1}} y^{d_{1}} \cdots x^{c_{t}} y^{d_{t}}$ with $c_{i}, d_{i} \geq 1$ for $1 \leq i \leq t$. This implies that the number of entries which are at least 2 in $\mathbf{a}$ is equal to the number of entries which are at least 2 in $\mathbf{b}$. In other words, $l(\mathbf{a}-1)=l(\mathbf{b}-1)$. Conversely, if $(\mathbf{a}, \mathbf{b}) \in \mathcal{P}(n, a, b)$ with $l(\mathbf{a})+l(\mathbf{b})=n$, $l(\mathbf{a}-1)=l(\mathbf{b}-1), \mathbf{a}-1=\left(c_{1}, \cdots, c_{t}\right)$ and $\mathbf{b}-1=\left(d_{1}, \cdots, d_{t}\right)$, then set

$$
(A, B)=M\left(x^{c_{1}} y^{d_{1}} \cdots x^{c_{t}} y^{d_{t}}, \lambda\right)
$$

Clearly, we have $p(A)=\mathbf{a}, p(B)=\mathbf{b}$, and $(A, B)$ (and therefore also $(\mathbf{a}, \mathbf{b}))$ is regular. This finishes the proof.

Altogether, we get that for a regular $(A, B) \in \mathrm{V}(n, a, b)$ the following are equivalent:

- $\operatorname{dim} \operatorname{top}(A, B)=p$;

- $\operatorname{dim} \operatorname{soc}(A, B)=p$;

- $\operatorname{dim}(\operatorname{Ker}(A) \cap \operatorname{Ker}(B))=p$; 
- $\operatorname{dim}(\operatorname{Im}(A) \cap \operatorname{Im}(B))=p$;

- $l(p(A)-1)=p$

- $l(p(B)-1)=p$.

Example. Let $(A, B)=M(x x y x y, \lambda)$. Then $p(A)=(3,2)$ and $p(B)=(2,2,1)$. Thus, $l(p(A)-1)=l(2,1)=2$ and $l(p(B)-1)=l(1,1)=2$. It is also clear that $M(x x y x y, \lambda)$ has a 2-dimensional socle and a 2-dimensional top. As an illustration, see Figure 5.

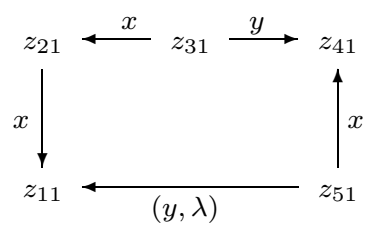

FIG. 5. The band module $M(x x y x y, \lambda)$

The next lemma follows directly from the construction of projective covers of indecomposable $\Lambda$-modules. These covers are easy to construct.

Lemma 5.3. Assume that $\mathcal{S}(L) \subset \mathrm{V}(n, a, b)$ contains a regular element $(A, B)$. Then $L$ is a direct sum of $n$ indecomposable modules, and exactly $n-\operatorname{dim} \operatorname{top}(A, B)$ of these are projective.

A $\Lambda$-module is called a diamond module if it is isomorphic to $M\left(x^{i} y^{j}, \lambda\right)$ for some $1 \leq i \leq a-1$ and $1 \leq j \leq b-1$. Thus the diamond modules are the band modules with simple top (and therefore also with simple socle). We now associate to any regular element $(\mathbf{a}, \mathbf{b})$ a diamond family $\mathcal{F}(\mathbf{a}, \mathbf{b})$ which consists of direct sums of diamond modules.

Let $(\mathbf{a}, \mathbf{b}) \in \mathcal{P}(n, a, b)$ be regular. Thus, $l(\mathbf{a}-1)=l(\mathbf{b}-1)$ by Proposition 1.3. Assume that $\mathbf{a}-1=\left(c_{1}, \cdots, c_{t}\right)$ and $\mathbf{b}-1=\left(d_{1}, \cdots, d_{t}\right)$. Let

$$
\mathcal{F}(\mathbf{a}, \mathbf{b})=\mathcal{F}\left(x^{c_{1}} y^{d_{t}}, x^{c_{2}} y^{d_{t-1}}, \cdots, x^{c_{t}} y^{d_{1}}\right) .
$$

Thus every module in $\mathcal{F}(\mathbf{a}, \mathbf{b})$ is isomorphic to

$$
\bigoplus_{i=1}^{t} M\left(x^{c_{i}} y^{d_{t-i+1}}, \lambda_{i}\right)
$$

for some pairwise different $\lambda_{i}$. For example, a module in

$$
\mathcal{F}((4,3,2,1),(3,2,2,1,1,1))
$$

looks as in Figure 6, where the points are just the basis vectors of the module. Note that $\mathcal{F}(\mathbf{a}, \mathbf{b}) \subset \Delta(\mathbf{a}, \mathbf{b})$. 

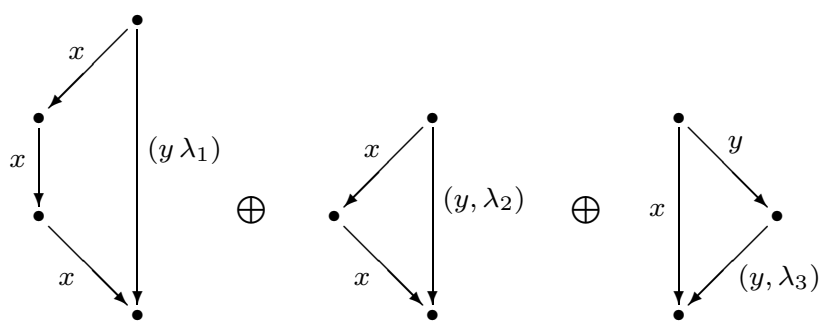

FIG. 6. An element in $\mathcal{F}((4,3,2,1),(3,2,2,1,1,1))$

Proposition 5.4. If $(\mathbf{a}, \mathbf{b}) \in \mathcal{P}(n, a, b)$ is regular, then $\mathcal{F}(\mathbf{a}, \mathbf{b})$ is dense in $\Delta(\mathbf{a}, \mathbf{b})$ and has dimension

$$
n^{2}-\sum_{i=1}^{r} m_{i}^{2}-\sum_{i=1}^{s} n_{i}^{2}+l(\mathbf{a}-1)^{2}
$$

where $(\mathbf{a}-1)^{*}=\left(m_{1}, \cdots, m_{r}\right)$ and $(\mathbf{b}-1)^{*}=\left(n_{1}, \cdots, n_{s}\right)$. In particular, $\Delta(\mathbf{a}, \mathbf{b})$ is irreducible.

Proof. Let $(A, B) \in \Delta(\mathbf{a}, \mathbf{b})$ be regular. Thus $(A, B)$ is in some stratum $\mathcal{S}(L)$ with $L$ a direct sum of $n$ indecomposable modules, and exactly $n-l(\mathbf{a}-1)$ of them are projective, see Lemma 5.3. By Lemma 4.3 we get

$$
\operatorname{dim} \operatorname{Hom}(L, \Lambda)=n(d-1)+l(\mathbf{a}-1)
$$

where $d=\operatorname{dim}(\Lambda)$. Assume $\mathbf{a}-1=\left(c_{1}, \cdots, c_{t}\right)$ and $\mathbf{b}-1=\left(d_{1}, \cdots, d_{t}\right)$. By Proposition 1.3 each module in $\Delta(\mathbf{a}, \mathbf{b})$ is isomorphic to a direct sum of band modules, and one checks easily that $L=(E, F)$ with

$$
p(E)=\left(e_{1}, \cdots, e_{t}\right)=\left(a-c_{t}-1, a-c_{t-1}-1, \cdots, a-c_{1}-1\right)
$$

and

$$
p(F)=\left(f_{1}, \cdots, f_{t}\right)=\left(b-d_{t}-1, b-d_{t-1}-1, \cdots, b-d_{1}-1\right) .
$$

Define

$$
L(\mathbf{a}, \mathbf{b})=\Lambda^{n-t} \oplus \bigoplus_{i=1}^{t} M\left(x^{e_{i}} y^{f_{t-i+1}}\right) .
$$

We apply a sequence of flip degenerations to $L$ and get

$$
L(\mathbf{a}, \mathbf{b}) \leq_{\operatorname{deg}} L
$$

with

$$
\operatorname{dim} \operatorname{Hom}(L(\mathbf{a}, \mathbf{b}), \Lambda)=\operatorname{dim} \operatorname{Hom}(L, \Lambda) .
$$

Then Theorem 2.1, (3) yields that the stratum $\mathcal{S}(L(\mathbf{a}, \mathbf{b}))$ is dense in $\Delta(\mathbf{a}, \mathbf{b})$. By $2.1,(1)$ we get that $\Delta(\mathbf{a}, \mathbf{b})$ is irreducible. Observe that

$$
\mathcal{F}(\mathbf{a}, \mathbf{b}) \subset \mathcal{S}(L(\mathbf{a}, \mathbf{b})) .
$$


We have

$$
\operatorname{dim} \mathcal{F}(\mathbf{a}, \mathbf{b})=n^{2}-\sum_{i=1}^{r} m_{i}^{2}-\sum_{i=1}^{s} n_{i}^{2}+l(\mathbf{a}-1)^{2}
$$

where $(\mathbf{a}-1)^{*}=\left(m_{1}, \cdots, m_{r}\right)$ and $(\mathbf{b}-1)^{*}=\left(n_{1}, \cdots, n_{s}\right)$. This follows from Lemma 3.5, the dimension formula for orbits and [Kr]. Using the dimension formula in Theorem 2.1, (1) and applying [CB] we get

$$
\operatorname{dim} \mathcal{S}(L(\mathbf{a}, \mathbf{b}))=\operatorname{dim} \mathcal{F}(\mathbf{a}, \mathbf{b})
$$

This implies that $\mathcal{F}(\mathbf{a}, \mathbf{b})$ is dense in $\Delta(\mathbf{a}, \mathbf{b})$.

Thus, from the above proposition we get the remarkable result that the diamond families form a dense subset in the set of all regular elements in $\mathrm{V}(n, a, b)$.

\section{The nilpotent case}

The following is easy to prove.

Lemma 6.1. For $u \in\{x, y\}$ and strings $C$ and $D$ the following hold:

(1) If $C u D$ is a string, then

$$
M(C) \oplus M(D) \in \overline{\mathcal{O}(M(C u D))} ;
$$

(2) If $C u$ is a band, then

$$
M(C) \in \overline{\mathcal{F}(C u)}=\overline{\mathcal{F}(u C)}
$$

Lemma 6.2. If $1 \leq i \leq a-1,1 \leq j \leq b-1$, and $l \geq 0$ such that $j+l+1 \leq b-1$, then

$$
M\left(x^{i} y^{j}, \lambda\right) \oplus M\left(y^{l}\right) \in \overline{\mathcal{F}\left(x^{i} y^{j+l+1}\right)} .
$$

Proof. There exists a short exact sequence

$$
0 \longrightarrow M\left(x^{i} y^{j}, \lambda\right) \longrightarrow M\left(y^{j+l} x^{i}\right) \longrightarrow M\left(y^{l}\right) \longrightarrow 0 .
$$

Thus

$$
M\left(y^{j+l} x^{i}\right) \leq_{\operatorname{deg}} M\left(x^{i} y^{j}, \lambda\right) \oplus M\left(y^{l}\right) .
$$

Then we use Lemma 6.1, (2).

Lemma 6.3. Let $(C, D) \in \mathrm{V}(n, n, n)$ with $\operatorname{rk}(C)+\operatorname{rk}(D)<n$ and $\operatorname{rk}(D)<n-1$. Then $(C, D)$ is contained in the closure of

$$
\{(A, B) \in \mathrm{V}(n, n, n) \mid \operatorname{rk}(A)=\operatorname{rk}(C), \operatorname{rk}(B)=\operatorname{rk}(D)+1\} .
$$


Proof. Set

$$
\mathcal{C}=\{(A, B) \in \mathrm{V}(n, n, n) \mid \operatorname{rk}(A)=\operatorname{rk}(C), \operatorname{rk}(B)=\operatorname{rk}(D)+1\},
$$

and let $s=n-\operatorname{rk}(C)-\operatorname{rk}(D)$. Thus $(C, D)$ is isomorphic to a module

$$
M \oplus \bigoplus_{i=1}^{s} M\left(C_{i}\right)
$$

where $M=0$ or $M$ is a direct sum of band modules. There are three cases to consider: First, if $s \geq 2$, then

$$
(C, D) \in \overline{\mathcal{O}\left(M \oplus M\left(C_{1} y C_{2}\right) \oplus M\left(C_{3}\right) \oplus \cdots \oplus M\left(C_{s}\right)\right)} \subseteq \overline{\mathcal{C}} .
$$

Second, if $s=1$ and $C_{1} \neq y^{l}$ for some $l \geq 0$, then

$$
(C, D) \in \overline{M \oplus \mathcal{F}\left(C_{1} y\right)} \subseteq \overline{\mathcal{C}} .
$$

Finally, assume that $s=1$ and $C_{1}=y^{l}$ for some $l \geq 0$. Since $\operatorname{rk}(D)<n-1$, this implies $l<n-1$ and thus $M \neq 0$. Using Proposition 5.4 we can assume without loss of generality that $M$ is a direct sum of diamond modules. Let $M\left(x^{i} y^{j}, \lambda\right)$ be one of these direct summands, thus $M=M^{\prime} \oplus M\left(x^{i} y^{j}, \lambda\right)$ for some $M^{\prime}$. Then we use Lemma 6.2 and get

$$
(C, D) \in \overline{M^{\prime} \oplus \mathcal{F}\left(x^{i} y^{j+l+1}\right)} \subseteq \overline{\mathcal{C}} .
$$

Note that we used several times our assumption $a=b=n$ by assuming that certain words in $x$ and $y$ are actually strings, i.e. that they do not contain subwords of the form $x^{a}$ or $y^{b}$. This finishes the proof.

Corollary 6.4. Let $(A, B) \in \mathrm{V}(n, n, n)$ with $\operatorname{rk}(A) \leq n-i$ and $\operatorname{rk}(B) \leq i$. Then $(A, B)$ is contained in the closure of

$$
\{(A, B) \in \mathrm{V}(n, n, n) \mid \operatorname{rk}(A)=n-i, \operatorname{rk}(B)=i\} .
$$

Lemma 6.5. If $u_{1}, u_{2}, v_{1}, v_{2} \geq 1, u_{1}+u_{2} \leq a-1$ and $v_{1}+v_{2} \leq b-1$, then

$$
M\left(x^{u_{1}+u_{2}} y^{v_{1}+v_{2}},-\lambda_{1} \lambda_{2}\right) \leq_{\operatorname{deg}} M\left(x^{u_{1}} y^{v_{1}}, \lambda_{1}\right) \oplus M\left(x^{u_{2}} y^{v_{2}}, \lambda_{2}\right) .
$$

Proof. It is straightforward to construct a short exact sequence

$$
0 \longrightarrow M\left(x^{u_{1}} y^{v_{1}}, \lambda_{1}\right) \longrightarrow M\left(x^{u_{1}+u_{2}} y^{v_{1}+v_{2}},-\lambda_{1} \lambda_{2}\right) \longrightarrow M\left(x^{u_{2}} y^{v_{2}}, \lambda_{2}\right) \longrightarrow 0 .
$$

Proof of Theorem 1.1. Let $(\mathbf{a}, \mathbf{b}) \in \mathcal{P}(n, n, n)$ be regular with

$$
\mathbf{a}-1=\left(c_{1}, \cdots, c_{t}\right)
$$

and

$$
\mathbf{b}-1=\left(d_{1}, \cdots, d_{t}\right)
$$


The diamond family $\mathcal{F}(\mathbf{a}, \mathbf{b})$ is dense in $\Delta(\mathbf{a}, \mathbf{b})$ by Proposition 5.4 , and each module in $\mathcal{F}(\mathbf{a}, \mathbf{b})$ is of the form

$$
\bigoplus_{j=1}^{t} M\left(x^{c_{j}} y^{d_{t-j+1}}, \lambda_{j}\right)
$$

for some $\lambda_{j}$. Since $a=b=n$, we know that $x^{n-i} y^{i}$ is a string for all $1 \leq i \leq n-1$. Now we use Lemma 6.5 and get that

$$
\mathcal{F}(\mathbf{a}, \mathbf{b}) \subset \Delta(\mathbf{a}, \mathbf{b}) \subset \overline{\mathcal{F}\left(x^{n-i} y^{i}\right)},
$$

where

$$
n-i=\sum_{j=1}^{t} c_{j}
$$

and

$$
i=\sum_{j=1}^{t} d_{j}
$$

This implies

$$
\{(A, B) \in \mathrm{V}(n, n, n) \mid \operatorname{rk}(A)=n-i, \operatorname{rk}(B)=i\} \subset \overline{\mathcal{F}\left(x^{n-i} y^{i}\right)} .
$$

Then Corollary 6.4 implies

$$
\overline{\mathcal{F}\left(x^{n-i} y^{i}\right)}=\{(A, B) \in \mathrm{V}(n, n, n) \mid \operatorname{rk}(A) \leq n-i, \operatorname{rk}(B) \leq i\} .
$$

By Proposition 5.4 we get

$$
\operatorname{dim} \mathcal{F}\left(x^{n-i} y^{i}\right)=\operatorname{dim} \overline{\mathcal{F}\left(x^{n-i} y^{i}\right)}=n^{2}-n+1 .
$$

This finishes the proof.

\section{Classification of regular irreducible components}

If $(\mathbf{a}, \mathbf{b}),(\mathbf{c}, \mathbf{d}) \in \mathcal{P}(n, a, b)$ with $\mathbf{a} \unlhd \mathbf{c}$ and $\mathbf{b} \unlhd \mathbf{d}$, then we write $(\mathbf{a}, \mathbf{b}) \unlhd(\mathbf{c}, \mathbf{d})$. This defines a partial order on $\mathcal{P}(n, a, b)$.

Lemma 7.1. If $(\mathbf{a}, \mathbf{b}),(\mathbf{c}, \mathbf{d}) \in \mathcal{P}(n, a, b)$ are regular with $(\mathbf{a}, \mathbf{b}) \unlhd(\mathbf{c}, \mathbf{d})$, then $l(\mathbf{a})=l(\mathbf{c})$ and $l(\mathbf{b})=l(\mathbf{d})$.

Proof. For all regular pairs $(\mathbf{e}, \mathbf{f})$ we have $l(\mathbf{e})+l(\mathbf{f})=n$. Since $\mathbf{a} \unlhd \mathbf{c}$, we have $l(\mathbf{a}) \geq l(\mathbf{c})$, and from $\mathbf{b} \unlhd \mathbf{d}$ we get $l(\mathbf{b}) \geq l(\mathbf{d})$. This implies $l(\mathbf{a})=l(\mathbf{c})$ and $l(\mathbf{b})=l(\mathbf{d})$.

The next lemma is a consequence of Proposition 5.1. 
Lemma 7.2. Let $(\mathbf{a}, \mathbf{b}),(\mathbf{c}, \mathbf{d}) \in \mathcal{P}(n, a, b)$. If

$$
\Delta(\mathbf{a}, \mathbf{b}) \cap \overline{\Delta(\mathbf{c}, \mathbf{d})} \neq \emptyset,
$$

then $(\mathbf{a}, \mathbf{b}) \unlhd(\mathbf{c}, \mathbf{d})$.

Let

$$
\begin{array}{r}
\mathcal{P}_{i, \text { reg }}^{p}=\mathcal{P}_{i, \text { reg }}^{p}(n, a, b)=\{(\mathbf{a}, \mathbf{b}) \in \mathcal{P}(n, a, b) \mid l(\mathbf{a})=i, l(\mathbf{b})=n-i, \\
l(\mathbf{a}-1)=p\},
\end{array}
$$

and

$$
\mathrm{V}_{i, \text { reg }}^{p}=\mathrm{V}_{i, \text { reg }}^{p}(n, a, b)=\bigcup_{(\mathbf{a}, \mathbf{b}) \in \mathcal{P}_{i, \text { reg }}^{p}} \Delta(\mathbf{a}, \mathbf{b}) .
$$

This implies

$$
\mathrm{V}_{i, \text { reg }}^{p}=\{(A, B) \in \mathrm{V}(n, a, b) \mid \operatorname{rk}(A)=n-i, \operatorname{rk}(B)=i, \operatorname{dim} \operatorname{top}(A, B)=p\} .
$$

In particular, $\mathrm{V}_{i, \mathrm{reg}}^{p}(n, a, b)$ is locally closed.

Proposition 7.3. Let $(\mathbf{a}, \mathbf{b}),(\mathbf{c}, \mathbf{d}) \in \mathcal{P}_{i, \text { reg }}^{p}(n, a, b)$. Then

$$
\Delta(\mathbf{a}, \mathbf{b}) \subset \overline{\Delta(\mathbf{c}, \mathbf{d})}
$$

if and only if $(\mathbf{a}, \mathbf{b}) \unlhd(\mathbf{c}, \mathbf{d})$.

Proof. If $(\mathbf{a}, \mathbf{b}) \unlhd(\mathbf{c}, \mathbf{d})$ does not hold, then we apply Lemma 7.2 and get

$$
\Delta(\mathbf{a}, \mathbf{b}) \cap \overline{\Delta(\mathbf{c}, \mathbf{d})}=\emptyset .
$$

Next, assume that $(\mathbf{a}, \mathbf{b}) \unlhd(\mathbf{c}, \mathbf{d})$ holds. By Lemma 5.3 each element in $V_{i, \text { reg }}^{p}$ belongs to some stratum of the form $\mathcal{S}(L)$ with $L$ a direct sum of $n$ indecomposables, and exactly $n-p$ of these are projective. Since $(\mathbf{a}, \mathbf{b}) \unlhd(\mathbf{c}, \mathbf{d})$ and $(\mathbf{a}, \mathbf{b}),(\mathbf{c}, \mathbf{d}) \in \mathcal{P}_{i, \text { reg }}^{p}(n, a, b)$, there exists a chain

$$
L(\mathbf{c}, \mathbf{d})=L_{1} \leq_{\operatorname{deg}} L_{2} \leq_{\operatorname{deg}} \cdots \leq_{\operatorname{deg}} L_{t}=L(\mathbf{a}, \mathbf{c})
$$

of box move degenerations between index modules such that $\operatorname{dim} \operatorname{Hom}\left(L_{i}, \Lambda\right)$ is constant for all $L_{i}$ in this chain. Now we use the same arguments as in the proof of Proposition 5.4, and finally we apply Theorem 2.1, (3). This finishes the proof.

An element $(\mathbf{a}, \mathbf{b}) \in \mathcal{P}_{i, \text { reg }}^{p}(n, a, b)$ is called $(i, p)$-maximal if it is maximal in $\mathcal{P}_{i, \text { reg }}^{p}(n, a, b)$ with respect to the partial order $\unlhd$. Clearly, each non-empty $\mathcal{P}_{i, \text { reg }}^{p}(n, a, b)$ contains a unique $(i, p)$-maximal element.

It follows easily that an element $(\mathbf{a}, \mathbf{b}) \in \mathcal{P}_{i, \text { reg }}^{p}(n, a, b)$ is $(i, p)$-maximal if and only if the following hold:

- a has at most one entry different from 1,2 and $a$; 
- b has at most one entry different from 1,2 and $b$.

As a consequence of Propositions 5.4 and 7.3 we get the following:

Corollary 7.4. The set $\mathrm{V}_{i, \mathrm{reg}}^{p}(n, a, b)$ is locally closed and irreducible, and if it is non-empty, then it contains $\mathcal{F}(\mathbf{a}, \mathbf{b})$ as a dense subset, where $(\mathbf{a}, \mathbf{b})$ is the unique $(i, p)$-maximal element in $\mathcal{P}_{i, \mathrm{reg}}^{p}(n, a, b)$.

Proof of Theorem 1.4. We characterize the $(i, p)$-maximal elements $(\mathbf{a}, \mathbf{b})$ such that the closure of $\Delta(\mathbf{a}, \mathbf{b})$ is an irreducible component. By the preceding results, these are then all regular irreducible components. Assume that $(\mathbf{a}, \mathbf{b})$ is $(i, p)$-maximal. Thus

$$
\mathbf{a}-1=\left((a-1)^{p-r-1}, a-v-1,1^{r}\right)
$$

and

$$
\mathbf{b}-1=\left((b-1)^{p-s-1}, b-w-1,1^{s}\right)
$$

where $0 \leq v \leq a-2,0 \leq w \leq b-2,0 \leq r, s \leq p-1, v=0 \Rightarrow r=0$ and $w=0 \Rightarrow s=0$. By Corollary 7.4 we have

$$
\overline{\mathcal{F}(\mathbf{a}, \mathbf{b})}=\overline{\Delta(\mathbf{a}, \mathbf{b})}=\overline{\mathrm{V}_{i, \mathrm{reg}}^{p}} .
$$

We claim that the closure of $\mathcal{F}(\mathbf{a}, \mathbf{b})$ is an irreducible component if and only if $r+s+1 \leq p$.

First, let $r+s+1>p$. This implies that there exist $u_{1}, u_{2}, v_{1}, v_{2} \geq 1$ such that each module in $\mathcal{F}(\mathbf{a}, \mathbf{b})$ has a direct summand isomorphic to

$$
M\left(x^{u_{1}} y^{v_{1}}, \lambda_{1}\right) \oplus M\left(x^{u_{2}} y^{v_{2}}, \lambda_{2}\right)
$$

where $u_{1}+u_{2} \leq a-1$ and $v_{1}+v_{2} \leq b-1$. Now we apply Lemma 6.5 and see that $\mathcal{F}(\mathbf{a}, \mathbf{b})$ is contained in the closure of some other family $\mathcal{F}(\mathbf{c}, \mathbf{d})$. In particular, the closure of $\mathcal{F}(\mathbf{a}, \mathbf{b})$ cannot be an irreducible component. This proves one direction of the statement.

Second, assume that $r+s+1 \leq p$. Since the function $\operatorname{rk}(-)$ is lower semicontinuous, $\mathrm{V}_{i \text {,reg }}^{p}$ cannot be contained in the closure of some $\mathrm{V}_{j \text {,reg }}^{q}$ with $i \neq j$. It is also clear that $\mathrm{V}_{i, \text { reg }}^{p}$ cannot be in the closure of $\mathrm{V}_{j \text {,reg }}^{q}$ if $p<q$. Because in that case, we have

$$
\operatorname{dim} \operatorname{Hom}(M, S)=p<q=\operatorname{dim} \operatorname{Hom}(N, S)
$$

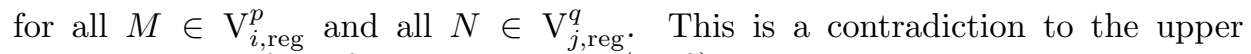
semicontinuity of the function $\operatorname{dim} \operatorname{Hom}(-, S)$.

Thus, assume that $i=j, p>q$ and $r+s+1 \leq p$. Then the dimension formula in Proposition 5.4 yields

$$
\operatorname{dim} \mathrm{V}_{i, \mathrm{reg}}^{p} \geq \operatorname{dim} V_{j, \mathrm{reg}}^{q} .
$$

Again this implies that $\mathrm{V}_{i \text {,reg }}^{p}$ cannot be in the closure of $\mathrm{V}_{j \text {,reg. }}^{q}$. Thus the closure of $\mathrm{V}_{i, \text { reg }}^{p}$ must be an irreducible component. Finally, note that $l(\mathbf{a}-1) \leq \mid a \in$ $\mathbf{a}|+| b \in \mathbf{b} \mid+1$ if and only if $r+s+1 \leq p$. This finishes the proof. 
Proof of Theorem 1.2. Let $\mathbf{a} \in \mathcal{P}(n, n)$ be a partition of $n$. If $\mathbf{a}=(1, \cdots, 1)$, then $\Delta(\mathbf{a})$ is the union of the orbits of $n$-dimensional modules of the form

$$
\bigoplus_{i \geq 0} M\left(y^{i}\right)^{m_{i}}
$$

and $\mathcal{O}\left(M\left(y^{n-1}\right)\right)$ is dense in $\Delta(\mathbf{a})$. Thus $\Delta(\mathbf{a})$ is irreducible and

$$
\Delta(\mathbf{a}) \subset \overline{\mathcal{F}\left(x y^{n-1}\right)}=\overline{\Delta(2,1, \cdots, 1)} .
$$

Next, assume that $\mathbf{a} \neq(1, \cdots, 1)$. Thus $l(\mathbf{a})=i$ for some $1 \leq i \leq n-1$. Then there exists a unique maximal (with respect to $\unlhd$ ) partition $\mathbf{a}^{\circ}$ such that $\left(\mathbf{a}, \mathbf{a}^{\circ}\right)$ is regular. Namely, we have $\mathbf{a}^{\circ}=\left(r_{1}, \cdots, r_{n-i}\right)$ where

$$
r_{j}= \begin{cases}i-l(\mathbf{a}-1)+2 & \text { if } j=1, \\ 2 & \text { if } 2 \leq j \leq l(\mathbf{a}-1), \\ 1 & \text { otherwise. }\end{cases}
$$

Here we use our assumption $a=b=n$. By Proposition 7.3, we know that for any regular element $(\mathbf{a}, \mathbf{d})$ we have

$$
\Delta(\mathbf{a}, \mathbf{d}) \subset \overline{\Delta\left(\mathbf{a}, \mathbf{a}^{\circ}\right)} .
$$

Now, assume that $(\mathbf{a}, \mathbf{c})$ is non-regular with $\Delta(\mathbf{a}, \mathbf{c})$ non-empty. It follows from Lemma 6.3 that

$$
\Delta(\mathbf{a}, \mathbf{c}) \subset \overline{\Delta(\mathbf{a}, \mathbf{d})}
$$

for some regular $(\mathbf{a}, \mathbf{d})$.

This proves that $\Delta(\mathbf{a})$ has $\Delta\left(\mathbf{a}, \mathbf{a}^{\circ}\right)$ as a dense subset. Thus $\Delta(\mathbf{a})$ is irreducible.

Recall that for regular elements, $(\mathbf{a}, \mathbf{b}) \unlhd(\mathbf{c}, \mathbf{d})$ implies $l(\mathbf{a})=l(\mathbf{c})$, see Lemma 7.1. Using Lemma 7.2, we get that

$$
\Delta(\mathbf{a}) \subset \overline{\Delta(\mathbf{c})}
$$

implies $\mathbf{a} \unlhd \mathbf{c}$ and $l(\mathbf{a})=l(\mathbf{c})$. Conversely, assume $\mathbf{a} \unlhd \mathbf{c}$ and $l(\mathbf{a})=l(\mathbf{c})$. This implies $\mathbf{a}^{\circ} \unlhd \mathbf{c}^{\circ}$ and $l(\mathbf{a}-1) \geq l(\mathbf{c}-1)$. We get

$$
\Delta(\mathbf{a}) \subset \overline{\Delta(\mathbf{c})}
$$

by applying Lemma 6.5 in case $l(\mathbf{a}-1)>l(\mathbf{c}-1)$, or Proposition 7.3 in case $l(\mathbf{a}-1)=l(\mathbf{c}-1)$. This finishes the proof.

\section{Classification of non-regular irreducible components}

The classification of irreducible components of $\mathrm{V}(n, a, b)$ with $a<n$ and $b<n$ is less straightforward than for the case $a=b=n$. The main reason is that Corollary 6.4 does not hold in the general case. 
A module $M$ is semi-projective (respectively semi-injective) if it is isomorphic to

$$
\bigoplus_{i=1}^{t} M\left(C_{i}\right)
$$

where $C_{i}=x^{a-1} C_{i}^{\prime} y^{b-1}$ for some string $C_{i}^{\prime}$ and all $i$ (respectively $C_{i}=y^{b-1} C_{i}^{\prime} x^{a-1}$ for some string $C_{i}^{\prime}$ and all $i$ ). The next two statements are clear.

Lemma 8.1. If $M(C)$ is semi-projective and $M(D)$ semi-injective, then $C x D y$ is a band. Thus,

$$
M(C) \oplus M(D) \in \overline{\mathcal{F}(C x D y)} .
$$

Lemma 8.2. If $M(C)$ is not semi-projective and not semi-injective, then there exists some $u \in\{x, y\}$ such that $C u$ is a band. Thus,

$$
M(C) \in \overline{\mathcal{F}(C u)} .
$$

The next lemma is again a consequence of the construction of projective covers of string modules.

Lemma 8.3. Let $M \in \mathrm{V}(n, a, b)$ be a direct sum of $t$ string modules. If $M$ is semi-projective (respectively semi-injective), then $M$ is in some stratum $\mathcal{S}(L)$ with $\operatorname{dim} \operatorname{Hom}(L, S)=n-t$ (respectively $\operatorname{dim} \operatorname{Hom}(L, S)=n+t)$.

Lemma 8.4. If $M$ is a semi-projective module in $\mathrm{V}(n, a, b)$, then $M$ is not contained in the closure of the set of regular elements in $\mathrm{V}(n, a, b)$.

Proof. Let

$$
M=\bigoplus_{i=1}^{t} M\left(C_{i}\right)
$$

be semi-projective. We have $M \in \mathcal{S}(L)$ for some index module $L$. By Lemma 8.3 we have

$$
\operatorname{dim} \operatorname{Hom}(L, S)=n-t
$$

Now assume that $\mathcal{S}(L)$ is contained in the closure of some stratum $\mathcal{S}(L(\mathbf{a}, \mathbf{b}))$ with $(\mathbf{a}, \mathbf{b})$ regular. So $L(\mathbf{a}, \mathbf{b}) \leq_{\operatorname{deg}} L$. Since $(\mathbf{a}, \mathbf{b})$ is regular, we get

$$
\operatorname{dim} \operatorname{Hom}(\mathcal{S}(L(\mathbf{a}, \mathbf{b}), S)=n .
$$

This is a contradiction because the function $\operatorname{dim} \operatorname{Hom}(-, S)$ is upper semicontinuous.

Lemma 8.4 enables us to determine when all irreducible components of $\mathrm{V}(n, a, b)$ are regular, i.e we can prove Proposition 1.5. 
Proof of Proposition 1.5. If $n \leq a+b-2$ or $n=a+b$, then there are no semiprojective or semi-injective modules. So Lemma 8.2 implies the result. For the other direction, it is sufficient to construct for each $n \geq a+b+1$ and for $n=a+b-1$ an $n$-dimensional semi-projective module. We leave this as an easy exercise to the reader. Then Lemma 8.4 yields the result.

Lemma 8.5. Let $M(C)$ be semi-projective, and let $B$ be a band of the form $x^{c} y^{d}$. Then there exists a semi-projective string module $M(E)$ such that

$$
M(E) \leq_{\operatorname{deg}} M(C) \oplus M(B, \lambda) .
$$

Proof. Let $B=x^{c} y^{d}$ for some $1 \leq c \leq a-1$ and $1 \leq d \leq b-1$, and let

$$
C=x^{c_{1}} y^{d_{1}} \cdots x^{c_{t}} y^{d_{t}}
$$

where $1 \leq c_{i} \leq a-1$ and $1 \leq d_{i} \leq b-1$ for all $i, c_{1}=a-1$ and $d_{t}=b-1$. Note that $M(C)$ is semi-projective. Let $m$ be the maximal $i$ such that one of the following hold:

(1) $c_{i}>c$;

(2) $c_{i}=c$ and $d_{i-1}<d$;

(3) $i=1$.

First, we assume that there exists some $i \geq m$ such that $d_{i}<d$. Note that this implies $i<t$. Then it follows from the definition of $m$ that $c_{i+1}<c$. Define

$$
E=x^{c_{1}} y^{d_{1}} \cdots y^{d_{i}} x^{c} y^{d} x^{c_{i+1}} \cdots x^{c_{t}} y^{d_{t}} .
$$

Now it is easy to construct a short exact sequence

$$
0 \longrightarrow M(C) \longrightarrow M(E) \longrightarrow M(B, \lambda) \longrightarrow 0 .
$$

This implies $M(E) \leq_{\operatorname{deg}} M(C) \oplus M(B, \lambda)$.

Second, we consider the case $d_{i} \geq d$ for all $i \geq m$. Let $l$ be maximal such that

$$
C=x^{c_{1}} y^{d_{1}} \cdots y^{d_{m-1}} x^{c_{m}-c}\left(x^{c} y^{d}\right)^{l} D
$$

for some string $D$. Define

$$
E=x^{c_{1}} y^{d_{1}} \cdots y^{d_{m-1}} x^{c_{m}-c}\left(x^{c} y^{d}\right)^{l+1} D .
$$

Again, one can construct a short exact sequence

$$
0 \longrightarrow M(C) \longrightarrow M(E) \longrightarrow M(B, \lambda) \longrightarrow 0
$$

which implies $M(E) \leq_{\operatorname{deg}} M(C) \oplus M(B, \lambda)$. This finishes the proof.

Let $\mathcal{P}_{n}$ (respectively $\mathcal{I}_{n}$ ) be the set of all semi-projective (respectively semiinjective) modules in $\mathrm{V}(n, a, b)$. Observe that $\mathcal{P}_{n}$ and $\mathcal{I}_{n}$ contain only finitely many isomorphism classes of modules. The next corollary follows from Proposition 5.4, Lemmas 8.1, 8.2 and 8.5. 
Corollary 8.6. Each non-regular irreducible component of $\mathrm{V}(n, a, b)$ contains a dense orbit $\mathcal{O}$ with $\mathcal{O} \subset \mathcal{P}_{n} \cup \mathcal{I}_{n}$.

Note that the duality $\mathrm{D}=\operatorname{Hom}_{K}(-, K)$ induces an isomorphism

$$
\begin{gathered}
\theta: \mathrm{V}(n, a, b) \longrightarrow \mathrm{V}(n, a, b) \\
(A, B) \mapsto\left(A^{t}, B^{t}\right)
\end{gathered}
$$

where $M^{t}$ denotes the transpose of a matrix $M$. For example, if $(A, B)$ is isomorphic to $M(x x y)$, then $\theta(A, B)$ is isomorphic to $\mathrm{D} M(x x y)=M(y x x)$. The restriction of $\theta$ to $\mathcal{P}_{n}$ yields an isomorphism $\mathcal{P}_{n} \rightarrow \mathcal{I}_{n}$.

Lemma 8.7. Let $\mathcal{S}(L)$ be a stratum containing a semi-projective module, and let $\mathcal{S}(M)$ be a stratum containing a semi-injective module. Then

$$
\mathcal{S}(L) \not \overline{\mathcal{S}(M)} \text { and } \mathcal{S}(M) \nsubseteq \overline{\mathcal{S}(L)}
$$

Proof. By Lemma 8.3 we get

$$
\operatorname{dim} \operatorname{Hom}(L, S)=n-s
$$

and

$$
\operatorname{dim} \operatorname{Hom}(M, S)=n+t
$$

for some $s, t \geq 1$. This implies $M \not_{\mathrm{deg}} L$. Thus by Theorem 2.1, (2) the stratum $\mathcal{S}(L)$ cannot be contained in the closure of $\mathcal{S}(M)$. Next, assume that $\mathcal{S}(M)$ is contained in the closure of $\mathcal{S}(L)$. This implies that $\theta(\mathcal{S}(M))$ is contained in the closure of $\theta(\mathcal{S}(L))$ with $\theta(\mathcal{S}(M))$ containing a semi-projective and $\theta(\mathcal{S}(L))$ containing a semi-injective module. But this is a contradiction to the first part of the proof.

Up to now, we established the following: To classify all non-regular irreducible components of $\mathrm{V}(n, a, b)$, it is sufficient to decide which orbits in $\mathcal{P}_{n}$ are open.

Let $X$ be indecomposable and semi-projective, and assume that $X$ is contained in a stratum $\mathcal{S}(L)$. We want to determine when $\mathcal{O}(X)$ is open. We can assume that $L$ is flip minimal, otherwise we could use flip degenerations and Theorem 2.1 to show that $\mathcal{S}(L)$ and in particular $X$ is contained in the closure of some other stratum $\mathcal{S}(M)$ with $M$ being flip minimal.

Let $(\mathbf{a}, \mathbf{b}) \in \mathcal{P}(n, a, b)$ such that the following hold:

- $|a \in \mathbf{a}|,|b \in \mathbf{b}| \geq 1$;

- $l(\mathbf{a})+l(\mathbf{b})=n+1$

- $l(\mathbf{a}-1)=l(\mathbf{b}-1)$.

Let $\mathbf{a}-1=\left(c_{1}, \cdots, c_{t}\right), \mathbf{b}-1=\left(d_{1}, \cdots, d_{t}\right)$, and define

$$
P(\mathbf{a}, \mathbf{b})=M\left(x^{c_{1}} y^{d_{t}} x^{c_{2}} y^{d_{t-1}} \cdots y^{d_{2}} x^{c_{t}} y^{d_{1}}\right)
$$


and

$$
L(\mathbf{a}, \mathbf{b})=\Lambda^{n-t} \oplus \bigoplus_{i=2}^{t} M\left(x^{a-c_{i}-1} y^{b-d_{t-i+2}-1}\right) .
$$

Note that $L(\mathbf{a}, \mathbf{b})$ is an index module in $\mathcal{I}_{\Lambda}(n)$, and $P(\mathbf{a}, \mathbf{b})$ is semi-projective and contained in the stratum $\mathcal{S}(L(\mathbf{a}, \mathbf{b}))$. Observe also that $P(\mathbf{a}, \mathbf{b}) \in \Delta(\mathbf{a}, \mathbf{b})$. The index module $L(\mathbf{a}, \mathbf{b})$ is flip minimal. Furthermore, each flip minimal index module $L$ with $\mathcal{S}(L)$ containing an indecomposable semi-projective module is obtained in this way.

Lemma 8.8. Under the above assumptions, the orbit $\mathcal{O}(P(\mathbf{a}, \mathbf{b}))$ is dense in $\mathcal{S}(L(\mathbf{a}, \mathbf{b}))$.

Proof. Using the dimension formula in Theorem 2.1, (1) and Theorem 3.7, a straightforward calculation shows that

$$
\operatorname{dim} \mathcal{O}(P(\mathbf{a}, \mathbf{b}))=\operatorname{dim} \mathcal{S}(L(\mathbf{a}, \mathbf{b})) .
$$

Thus $\mathcal{O}(L(\mathbf{a}, \mathbf{b}))$ must be dense in the stratum $\mathcal{S}(L(\mathbf{a}, \mathbf{b}))$.

As a consequence of the above results we get the following:

Lemma 8.9. The orbit $\mathcal{O}(P(\mathbf{a}, \mathbf{b}))$ is open if and only if there is no module $P(\mathbf{c}, \mathbf{d})$ with $P(\mathbf{c}, \mathbf{d})<_{\operatorname{deg}} P(\mathbf{a}, \mathbf{b})$.

Lemma 8.10. Let $(\mathbf{a}, \mathbf{b}),(\mathbf{c}, \mathbf{d}) \in \mathcal{P}(n, a, b)$ such that

- $|a \in \mathbf{a}|,|b \in \mathbf{b}|,|a \in \mathbf{c}|,|b \in \mathbf{d}| \geq 1$

- $l(\mathbf{a})+l(\mathbf{b})=l(\mathbf{c})+l(\mathbf{d})=n+1$

- $l(\mathbf{a}-1)=l(\mathbf{b}-1)$ and $l(\mathbf{c}-1)=l(\mathbf{d}-1)$.

Then the following hold:

(1) If $P(\mathbf{c}, \mathbf{d}) \leq_{\operatorname{deg}} P(\mathbf{a}, \mathbf{b})$, then $(\mathbf{a}, \mathbf{b}) \unlhd(\mathbf{c}, \mathbf{d})$;

(2) If $(\mathbf{a}, \mathbf{b}) \unlhd(\mathbf{c}, \mathbf{d})$ and $l(\mathbf{a}-1)=l(\mathbf{c}-1)$, then $P(\mathbf{c}, \mathbf{d}) \leq_{\operatorname{deg}} P(\mathbf{a}, \mathbf{b})$.

Proof. The first part of the lemma is a direct consequence of Lemma 7.2. Next, one easily checks that the conditions $(\mathbf{a}, \mathbf{b}) \unlhd(\mathbf{c}, \mathbf{d})$ and $l(\mathbf{a}-1)=l(\mathbf{c}-1)$ allow a sequence of box move degenerations

$$
L(\mathbf{c}, \mathbf{d})=L_{1} \leq_{\operatorname{deg}} L_{2} \leq_{\operatorname{deg}} \cdots \leq_{\operatorname{deg}} L_{t}=L(\mathbf{a}, \mathbf{b})
$$

such that $\operatorname{dim} \operatorname{Hom}\left(L_{i}, \Lambda\right)=\operatorname{dim} \operatorname{Hom}\left(L_{1}, \Lambda\right)$ for all $i$. As before we use Theorem $2.1,(3)$ and get

$$
\mathcal{S}(L(\mathbf{a}, \mathbf{b})) \subseteq \overline{\mathcal{S}(L(\mathbf{c}, \mathbf{d}))} .
$$

Since $P(\mathbf{a}, \mathbf{b})$ and $P(\mathbf{c}, \mathbf{d})$ are dense in $\mathcal{S}(L(\mathbf{a}, \mathbf{b}))$ and $\mathcal{S}(L(\mathbf{c}, \mathbf{d}))$, respectively, this implies $P(\mathbf{c}, \mathbf{d}) \leq_{\operatorname{deg}} P(\mathbf{a}, \mathbf{b})$. This finishes the proof. 
Theorem 8.11 (Classification of open orbits). Let $X$ be an indecomposable $\Lambda$ module. Then $\mathcal{O}(X)$ is open in $\mathrm{V}(n, a, b)$ if and only if $X$ is isomorphic to $M(C)$ or $\mathrm{D} M(C)$ where $C$ is of one of the following forms:

(1)

$$
C=\left(x^{a-1} y\right)^{r}\left(x^{a-1} y^{b-1}\right)^{s}\left(x y^{b-1}\right)^{t}
$$

(2)

where $r, s, t \geq 0, r+s \geq 1$ and $s+t \geq 1$;

$$
C=\left(x^{a-1} y\right)^{r}\left(x^{a-1} y^{i}\right)^{\alpha}\left(x^{a-1} y^{b-1}\right)^{s}\left(x^{j} y^{b-1}\right)^{\beta}\left(x y^{b-1}\right)^{t}
$$

where $r, s, t \geq 0,2 \leq i \leq b-2,2 \leq j \leq a-2,0 \leq \alpha, \beta \leq 1, \alpha+\beta \geq 1$, $r+\alpha+s \geq 1$ and $s+\beta+t \geq 1$;

(3)

$$
C=\left(x^{a-1} y\right)^{r} x^{i} y^{j}\left(x y^{b-1}\right)^{t}
$$

where $r, t \geq 1,1 \leq i \leq a-2$ and $1 \leq j \leq b-2$.

The open orbits in $\mathrm{V}(n, a, b)$ are exactly the orbits of the form

$$
\mathcal{O}\left(\bigoplus_{i \in I} M\left(C_{i}\right)\right)
$$

with $\mathcal{O}\left(M\left(C_{i}\right)\right)$ open and $\operatorname{Ext}^{1}\left(M\left(C_{i}\right), M\left(C_{j}\right)\right)=0$ for all $i \neq j$ in $I$.

If a string $C$ belongs to one of the sets (1), (2) or (3) as defined in the theorem, then we say that $C$ is of type (1), (2) or (3), respectively.

Proof. We classify the open orbits $\mathcal{O}(X)$ with $X$ indecomposable. By Lemma 8.2 we know that $X$ has to be semi-projective or semi-injective. By duality, we can assume without loss of generality that $X$ is semi-projective. As a consequence of Lemma 8.10, we can assume that $X=M(C)=P(\mathbf{a}, \mathbf{b})$ such that the following hold:

- a has at most one entry different from 1,2 and $a$;

- b has at most one entry different from 1,2 and $b$.

Now we proceed similar to the proof of Theorem 1.4. We can assume that $l(\mathbf{a})+$ $l(\mathbf{b})=n+1$,

$$
\mathbf{a}-1=\left((a-1)^{p-r-1}, a-v-1,1^{r}\right)
$$

and

$$
\mathbf{b}-1=\left((b-1)^{p-s-1}, b-w-1,1^{s}\right)
$$

where $0 \leq v \leq a-2,0 \leq w \leq b-2,0 \leq r, s \leq p-1, v=0 \Rightarrow r=0$ and $w=0 \Rightarrow s=0$. Then by using Theorem 3.7, we get

$$
\begin{aligned}
\operatorname{dim} \mathcal{O}(P(\mathbf{a}, \mathbf{b}))=n^{2}- & p^{2}-p-1-(a-v-2)(p-r)^{2} \\
& \quad-(b-w-2)(p-s)^{2}-v(p-r-1)^{2}-w(p-s-1)^{2} .
\end{aligned}
$$


By Lemma 8.9 the orbit of $P(\mathbf{a}, \mathbf{b})$ is open if and only if there is no $P(\mathbf{c}, \mathbf{d})$ with $P(\mathbf{c}, \mathbf{d})<_{\operatorname{deg}} P(\mathbf{a}, \mathbf{b})$.

If $r+s+1 \leq p$, then

$$
\operatorname{dim} \mathcal{O}(P(\mathbf{a}, \mathbf{b})) \geq \operatorname{dim} \mathcal{O}(P(\mathbf{c}, \mathbf{d}))
$$

for all $P(\mathbf{c}, \mathbf{d})$ with $(\mathbf{a}, \mathbf{b}) \unlhd(\mathbf{c}, \mathbf{d})$. This follows from the above dimension formula. So by Lemma 8.10 the orbit $\mathcal{O}(P(\mathbf{a}, \mathbf{b}))$ must be open. Observe that $C$ is of type (1), (2) or (3) if and only if $r+s+1 \leq p$.

Next, assume that $r+s+1>p$. By the definition of $r$ and $s$, it follows that $a, b \geq 3$ in this case. Then $C$ is of the form

$$
\left(x^{a-1} y\right)^{k}\left(x^{i} y\right)(x y)^{l}\left(x y^{j}\right)\left(x y^{b-1}\right)^{m}
$$

where $k, m \geq 1, l \geq 0,1 \leq i \leq a-2$ and $1 \leq j \leq b-2$. If $l=0$, then define

$$
E=\left(x^{a-1} y\right)^{k} x^{i+1} y^{j+1}\left(x y^{b-1}\right)^{m} \text {. }
$$

Otherwise, let

$$
E=\left(x^{a-1} y\right)^{k}\left(x^{i+1} y y\right)(x y)^{l-1}\left(x y^{j}\right)\left(x y^{b-1}\right)^{m} .
$$

In both cases, we get $M(E)<_{\operatorname{deg}} M(C)$. This is proved by constructing a Riedtmann sequence

$$
0 \longrightarrow M(C) \longrightarrow M(E) \oplus M(x y, 1) \longrightarrow M(x y, 1) \longrightarrow 0 .
$$

Thus, $\mathcal{O}(P(\mathbf{a}, \mathbf{b}))$ cannot be open. This finishes the classification of indecomposable $\Lambda$-modules whose orbit is open. The rest of the theorem follows from $[\mathrm{Z}$, Theorem 3].

For modules $X$ and $Y$ let $\operatorname{Hom}(X, Y)$ be the space $\operatorname{Hom}(X, Y)$ modulo the homomorphisms factoring through a projective module. By $\tau$ we denote the Auslander-Reiten translation. For indecomposable modules $X$ and $Y$ we have the Auslander-Reiten formula

$$
\operatorname{Ext}^{1}(X, Y) \cong \operatorname{DHom}\left(\tau^{-1} Y, X\right) .
$$

For the basics of Auslander-Reiten theory we refer to [ARS] or [Ri]. If $M(C)$ is a semi-projective string module, then define

$$
\tau^{-1} C=x^{a-1} y C x y^{b-1} .
$$

Note that $M\left(\tau^{-1} C\right)$ is also semi-projective. It is proved in [BR] that

$$
\tau^{-1} M(C)=M\left(\tau^{-1} C\right) .
$$

The next proposition is an application of the Auslander-Reiten formula and Theorem 3.7 .

Proposition 8.12. If $M(C)$ and $M(D)$ are semi-projective string modules, then the following are equivalent: 
(1) $\operatorname{Ext}^{1}(M(C), M(D))=0$;

(2) Each map $f_{a}$ with $a \in \mathcal{A}\left(\tau^{-1} D, C\right)$ factors through $M\left(x^{a-1} y^{b-1}\right)$.

For deciding whether a graph map factors through another string module, one uses the multiplicative behaviour of graph maps. Using this proposition and the previous theorem, it is now easy to compute the semi-projective modules whose orbit is open. Using duality, we get all open orbits. This completes the classification of irreducible components of the variety $\mathrm{V}(n, a, b)$.

Corollary 8.13. For an indecomposable $\Lambda$-module $X$ the following statements are equivalent:

(1) $\operatorname{Ext}^{1}(X, X)=0$;

(2) $X$ is isomorphic to a string module $M(C)$ or $\mathrm{D} M(C)$ with

$$
C=\left(x^{a-1} y\right)^{r}\left(x^{a-1} y^{b-1}\right)^{s}\left(x y^{b-1}\right)^{t}
$$

where $r, s, t \geq 0, r+s \geq 1$ and $s+t \geq 1$.

\section{Remarks and examples}

We list all irreducible components of $\mathrm{V}(n, 3,3)$ for $n \leq 12$. First, let us give the list of all regular irreducible components and their dimensions.

For each regular $(\mathbf{a}, \mathbf{b})$ we constructed a family $\mathcal{F}(\mathbf{a}, \mathbf{b})$ of modules which is dense in $\Delta(\mathbf{a}, \mathbf{b})$, see Proposition 5.4. Recall that these families are of the form $\mathcal{F}\left(\left(B_{1}, p_{1}\right), \cdots,\left(B_{m}, p_{m}\right)\right)$.

In Figure 7 we display the data $\left(B_{1}, p_{1}\right), \cdots,\left(B_{m}, p_{m}\right)$ in case the closure of the corresponding family is an irreducible component. If $p_{i}=1$, then we just write $B_{i}$ instead of $\left(B_{i}, p_{i}\right)$.

In Figure 8 we give a list of all open orbits and their dimensions. Recall that the closures of the open orbits are exactly the non-regular irreducible components. Remember also that the open orbits are orbits of certain semi-projective or semiinjective modules. For the sake of brevity we list only the strings $C_{i}$ occurring in their direct sum decomposition. For example $x x y y \oplus x x y y$ encodes the module $M(x x y y) \oplus M(x x y y)$. We only list the semi-projective modules whose orbits are open. Thus one has to add the same number of semi-injective modules to get all open orbits. Recall that there are no open orbits for $n \leq a+b-2=4$ and $n=a+b=6$.

Remark 1. If $\operatorname{Ext}^{1}(M, M)=0$ for some $\Lambda$-module $M$, then by Voigt's Lemma one gets that $\mathcal{O}(M)$ is open. The converse does not hold. The smallest example of this kind occurs for $n=9$ : Let $M=M($ xxyxyxyy) be in $\mathrm{V}(9,3,3)$. Then $\mathcal{O}(M)$ is open but $\operatorname{Ext}^{1}(M, M) \neq 0$. 


\begin{tabular}{c|c|c|c|r}
2 & 3 & 4 & 5 & 6 \\
\hline \hline$x y 3$ & $x x y 7$ & xxyy 13 & $x x y, x y 20$ & $(x x y, 2) 28$ \\
& xyy 7 & & $x y y, x y 20$ & $(x y y, 2) 28$ \\
& & & & $x x y, x y y 30$
\end{tabular}

\begin{tabular}{c|r|r}
\multicolumn{1}{c|}{7} & 8 & \multicolumn{1}{c|}{9} \\
\hline \hline xxyy, xxy 40 & $(x x y, 2), x y 51$ & $(x x y, 3) 63$ \\
xxyy, xyy 40 & $(x y y, 2), x y 51$ & $(x y y, 3) 63$ \\
& $(x x y y, 2) 52$ & $(x x y, 2), x y y 67$ \\
& xxy, xyy, xy 53 & $(x y y, 2)$, xxy 67
\end{tabular}

\begin{tabular}{r|r|r}
10 & 11 & \multicolumn{1}{c}{12} \\
\hline \hline$(x x y, 2)$, xxyy 81 & $(x x y, 3), x y 96$ & $(x x y, 4) 112$ \\
$(x y y, 2)$, xxyy 81 & $(x y y, 3), x y 96$ & $(x y y, 4) 112$ \\
$x x y y, x x y, x y y 83$ & $(x x y y, 2), x x y 99$ & $(x x y y, 3) 117$ \\
& $(x x y y, 2), x y y 99$ & $(x x y, 3), x y y 118$ \\
& $(x x y, 2), x y y, x y 100$ & $(x y y, 3), x x y 118$ \\
& $(x y y, 2), x x y, x y 100$ & $(x x y, 2),(x y y, 2) 120$
\end{tabular}

FIG. 7. The regular components of $\mathrm{V}(n, 3,3)$ for $n \leq 12$

\begin{tabular}{|c|c|c|c|c|c|}
\hline 5 & & 7 & \multicolumn{2}{|l|}{8} & 9 \\
\hline xxyy 20 & $x x y$ & cyy 40 & $\begin{array}{l}x x y y x y y \\
\text { xxyxxyy }\end{array}$ & $\begin{array}{l}52 \\
52\end{array}$ & $\begin{array}{rr}(x x y y)^{2} & 66 \\
x x y x y x y y & 66\end{array}$ \\
\hline 10 & & & 11 & & 12 \\
\hline $\begin{array}{r}x x y y \oplus x x y y \\
(x x y)^{2} x y y \\
x x y(x y y)^{2}\end{array}$ & $\begin{array}{l}80 \\
82 \\
82\end{array}$ & $\begin{array}{c}(x x y)^{2} \\
x x y y( \\
x x y x x ?\end{array}$ & $\begin{array}{ll}x x y y & 98 \\
x y y)^{2} & 98 \\
\text { xхyy } & 100\end{array}$ & & $\begin{array}{rl}x y \oplus x x y x y y & 117 \\
(x x y y)^{2} x y y & 118 \\
x x y(x x y y)^{2} & 118 \\
(x x y)^{2} x y x y y & 118 \\
x x y x y(x y y)^{2} & 118\end{array}$ \\
\hline
\end{tabular}

FIG. 8. The non-regular components of $\mathrm{V}(n, 3,3)$ for $n \leq 12$

Remark 2. Let $a=b=2$ and $n=3$. Then $\mathcal{O}(M(x y))$ and $\mathcal{O}(M(y x))$ are both open orbits, since $M(x y)$ is projective and $M(y x)$ is injective. In particular, $\Delta(2,1)$ and $\Delta((2,1),(2,1))$ are both not irreducible.

Remark 3. The Gelfand-Ponomarev algebra $\Lambda$ is a string algebra in the sense of [BR]. Similarly to Lemma 4.1 one can show that all string algebras are subfinite, and their index modules can be classified as in Lemma 4.2.

One should be able to classify the irreducible components of varieties of modules over many other string algebras in the same fashion as in this paper.

\section{References}

[ARS] M. Auslander, I. Reiten and S. Smalø, Representation theory of Artin algebras, Corrected reprint of the 1995 original. Cambridge Studies in Advanced Mathematics, 36. Cambridge 
University Press, Cambridge, 1997, xiv +425 pp.

[Bo] K. Bongartz, Some geometric aspects of representation theory, Algebras and modules I (Trondheim, 1996), 1-27, CMS Conf. Proc. 23, Amer. Math. Soc., Providence, RI, 1998.

[BR] M. C. R. Butler and C. M. Ringel, Auslander-Reiten sequences with few middle terms and applications to string algebras, Comm. Alg. 15 (1987), 145-179.

[CB] W. Crawley-Boevey, Maps between representations of zero-relation algebras, J. Algebra 126 (1989), 259-263.

[GP] I. M. Gelfand and V. A. Ponomarev, Indecomposable representations of the Lorentz group, Russian Math. Surveys 23 (1968), 1-58.

[Ge] M. Gerstenhaber, On dominance and varieties of commuting matrices, Ann. of Math. (2) 73 (1961), 324-348.

H] W. Hesselink, Singularities in the nilpotent scheme of a classical group, Trans. Amer. Math. Soc. 222 (1976), 1-32.

[K] H. Kraft, Geometric methods in representation theory, In: Representations of Algebras, Lecture Notes in Math. 944, Springer-Verlag, New York (1980), 180-257.

[Kr] H. Krause, Maps between tree and band modules, J. Algebra 137 (1991), 186-194.

[M] K. Morrison, The scheme of finite-dimensional representations of an algebra, Pacific J. Math. 91 (1980), 199-218.

[R] N. Richmond, A stratification for varieties of modules, Bull. London Math. Soc. 33 (2001), 565-577.

[Rie] C. Riedtmann, Degenerations for representations of quivers with relations, Ann. Sci. École Norm. Sup. (4) 19 (1986), 275-301.

[Ri] C. M. Ringel, Tame algebras and integral quadratic forms, Lecture Notes in Math. 1099, Springer-Verlag, New York, 1984, xiii+376 pp.

$[\mathrm{ZH}]$ B. Zimmermann-Huisgen, Predicting syzygies over monomial relations algebras, Manuscripta Math. 70 (1991), 157-182.

[Z] G. Zwara, Degenerations of finite-dimensional modules are given by extensions, Compositio Math. 121 (2000), 205-218.

Jan Schröer

Department of Pure Mathematics

University of Leeds

Leeds LS2 9JT

England

e-mail: jschroer@maths.leeds.ac.uk

(Received: January 11, 2000; revised version: February 5, 2003)

To access this journal online:

(2) http://www.birkhauser.ch 